\title{
MOLECULAR GAS IN EXTREME STAR-FORMING ENVIRONMENTS: THE STARBURSTS Arp 220 AND NGC 6240 AS CASE STUDIES
}

\author{
T. R. Greve ${ }^{1,2}$, P. P. PAPAdopoulos 33 , Y. GaO ${ }^{4}$, and S. J. E. RadFord ${ }^{2}$ \\ ${ }^{1}$ Max-Planck Institut für Astronomie, Königstuhl 17, 69117 Heidelberg, Germany; tgreve@mpia-hd.mpg.de \\ ${ }^{2}$ California Institute of Technology, Pasadena, CA 91125, USA; sradford@submm.caltech.edu \\ ${ }^{3}$ Argelander Institute for Astronomy, University of Bonn, Auf dem Hügel 71, 53121 Bonn, Germany; padeli@astro.uni-bonn.de \\ ${ }^{4}$ Purple Mountain Observatory, Chinese Academy of Sciences, Nanjing 210008, China; yugao@pmo.ac.cn \\ Received 2006 October 10; accepted 2008 September 15; published 2009 February 24
}

\begin{abstract}
We report single-dish multitransition measurements of the ${ }^{12} \mathrm{CO}, \mathrm{HCN}$, and $\mathrm{HCO}^{+}$molecular line emission as well as HNC $J=1-0$ and HNCO in the two ultraluminous IR galaxies Arp 220 and NGC 6240. Using this new molecular line inventory, in conjunction with existing data in the literature, we compiled the most extensive molecular line data sets to date for such galaxies. The many rotational transitions, with their different excitation requirements, allow the study of the molecular gas over a wide range of different densities and temperatures with significant redundancy, and thus allow good constraints on the properties of the dense gas in these two systems. The mass $\left(\sim(1-2) \times 10^{10} M_{\odot}\right)$ of dense gas $\left(\gtrsim 10^{5-6} \mathrm{~cm}^{-3}\right)$ found accounts for the bulk of their molecular gas mass, and is consistent with most of their IR luminosities powered by intense starbursts while self-regulated by $\mathrm{O}$, B star cluster radiative pressure onto the star-forming dense molecular gas. The highly excited $\mathrm{HCN}$ transitions trace a gas phase $\sim(10-100) \times$ denser than that of the subthermally excited $\mathrm{HCO}^{+}$ lines (for both galaxies). These two phases are consistent with an underlying density-size power law found for Galactic giant molecular clouds (but with a steeper exponent), with HCN lines tracing denser and more compact regions than $\mathrm{HCO}^{+}$. Whether this is true in IR-luminous, star-forming galaxies in general remains to be seen, and underlines the need for observations of molecular transitions with high critical densities for a sample of bright (U)LIRGs in the local universe - a task for which the HI-FI instrument on board Herschel is ideally suited to do.
\end{abstract}

Key words: galaxies: individual (Arp 220, NGC 6240) - galaxies: starburst - ISM: molecules

\section{INTRODUCTION}

A cardinal result in extragalactic astronomy over the last 20 years has been the discovery by IRAS of a population of local ultraluminous infrared galaxies (ULIRGs; Soifer et al. 1986; see also Sanders \& Mirabel 1996; Lonsdale et al. 2006). Although these galaxies are rare in the local universe, their extreme IR-luminosities $\left(L_{\mathrm{IR}} \sim 10^{12} L_{\odot}\right)$ and merger morphologies strongly suggest that they signify a highly active and important phase in galaxy evolution-a scenario which is corroborated by the rapid increase in their space density with redshift. Furthermore, ULIRGs-being gas-rich mergers harboring the most extreme star-forming conditions encountered in the local universe - are thought to re-enact the galaxyformation processes we are only barely able to glimpse in the distant universe. Although detailed studies of the interstellar medium (ISM) in protogalaxies at high redshifts will be possible with the new generation of centimeter/millimeter/submillimeter telescopes and receivers coming online within the next decade, our expectations of how such objects will look in millimeter/ submillimeter line emissions are ill-informed due to the dearth of similar data locally. Multiline studies of the low-intensity starbursts M 82 and NGC 253 have been made (Nguyen et al. 1992; Jackson et al. 1995; Paglione et al. 1997), but given that their star-formation rates (SFRs) are about three orders of magnitude lower than that of typical ULIRGs, and they may very well not be good guides for the state of the ISM in local ULIRGs and high- $z$ starbursts (Aalto et al. 1995; Papadopoulos \& Seaquist 1998; Downes \& Solomon 1998).

In contrast, local ULIRGs, owing to their extreme IR/CO brightness, are ideal targets for multiline studies of heavy rotor molecules such as $\mathrm{HCN}, \mathrm{CS}$, and $\mathrm{HCO}^{+}$. These lines are usually 5-30 times weaker than their CO J-level counterparts, making them currently impossible to detect in more distant galaxies and/or galaxies with lower SFRs. Second, their compact $\mathrm{H}_{2}$ distributions - as revealed by interferometric maps (e.g., Scoville et al. 1997; Tacconi et al. 1999)—allow us to collect the total line flux of these often weak lines with a single pointing. This is important since multibeam receivers, capable of observing many transitions of a variety of species within a reasonable amount of telescope time, are still lacking. These two factors, brightness and relatively compact molecular gas distributions, allow us to study the properties of the dense $\left(n\left(\mathrm{H}_{2}\right) \gtrsim 10^{4} \mathrm{~cm}^{-3}\right)$ gas phase in local ULIRGs in much greater detail than would otherwise be possible. This is of great importance, because the dense gas provides the immediate star-formation fuel in galaxies (Gao \& Solomon 2004a, 2004b; Wu et al. 2005). The possibility of a universal star-formation efficiency (SFE), as proposed by these studies, can now be checked in a much more powerful manner, since we can estimate the dense $\mathrm{H}_{2}$ mass using multiple lines rather than a single line such as $\mathrm{HCN} J=1-0$.

In the local universe, Arp 220 and NGC 6240 are ideally suited for studies of the dense gas in extreme starburst galaxies, as they are the two most studied (U)LIRGs to date. They have the best-sampled far-IR (FIR)/submillimeter spectral energy distributions (SEDs) of their class (e.g., Dopita et al. 2005), and the largest number of millimeter/submillimeter $\mathrm{CO}, \mathrm{HCN}, \mathrm{CS}$, and $\mathrm{HCO}^{+}$transitions already observed. Thus not only are their dust continuum SEDs well constrained, but it is also relatively straightforward to fill the gaps in their molecular line inventory. Being the nearest and best studied, Arp 220 is the ULIRG most frequently used as a template for high $-z$ studies of starbursts. The prominent active galactic nucleus (AGN) present in NGC 6240 (even double-AGN, Komossa et al. 2003, but not in Arp 220, 
e.g., Iwasawa et al. 2005) offers the possibility of studying the influence of an AGN on the molecular ISM, for example how $\mathrm{X}$-ray dissociation regions (XDRs) affect $\mathrm{H}_{2}$ cloud chemistry, significantly altering the $\mathrm{HCO}^{+}$and $\mathrm{HCN}$ abundances (Lepp \& Dalgarno 1996; Usero et al. 2004).

In this paper, we present single-dish observations of a number of molecular emission lines from Arp 220 and NGC 6240, which together with results from the literature allow us to compile a very large (and almost identical) molecular transition catalog for these two extreme starbursts. Armed with this unique catalog, and the large range of excitation conditions it can probe (with significant overlap/redudancy between $\mathrm{HCN}$ and CS lines) we use it to (1) set constraints on the physical properties of the dense molecular gas phase, (2) find the gas density probed per molecular species and its mass range, and (3) explore underlying SFEs, density-size hierarchies, and examine the species best suited for tracing the true star-forming phase.

This paper is outlined as follows: observations and results are described in Sections 2 and 3, respectively. Section 4 details the analysis of our observations, which includes deriving dense gas mass and SFEs based on the radiative transfer modeling of the observed lines. In Section 5, we discuss the implications of our findings in the context of upcoming missions such as Herschel. In the flat cosmology $\left(\Omega_{m}=0.27, \Omega_{\Lambda}=0.73\right.$, and $H_{0}=71 \mathrm{~km} \mathrm{~s}^{-1} \mathrm{Mpc}^{-3}$; Spergel et al. 2003), adopted throughout this paper the luminosity distances to Arp 220 and NGC 6240 are 77.5 and $105.5 \mathrm{Mpc}$, respectively.

\section{OBSERVATIONS}

The $15 \mathrm{~m}$ James Clerk Maxwell Telescope ${ }^{5}$ (JCMT) on Mauna Kea, Hawaii was used to observe a number of ${ }^{12} \mathrm{CO}$, ${ }^{13} \mathrm{CO}, \mathrm{HCN}, \mathrm{CS}$, and $\mathrm{HCO}^{+}$lines in Arp 220 and NGC 6240 (Table 1$)$. These observations were done in $\operatorname{good}\left(\tau_{225 \mathrm{GHz}} \lesssim\right.$ $0.035)$ to medium $\left(0.08 \lesssim \tau_{225 \mathrm{GHz}} \lesssim 0.12\right)$ weather conditions during several runs on the JCMT between 2003 January and 2004 December. The two JCMT receivers A3(211-279 GHz) and $B 3(312-370 \mathrm{GHz})$ were employed in order to observe all the lines, with the $B 3$ receiver tuned in the single-sideband (SSB) mode. In order to ensure baselines as flat as possible, the observations were done in fast beam-switching mode with a chop throw of $30^{\prime \prime}$ (AZ) and a chopping frequency of $1 \mathrm{~Hz}$. As backend we used the Digital Autocorrelation Spectrometer (DAS) in its widest, $\sim 1.8 \mathrm{GHz}\left(\sim 1100 \mathrm{~km} \mathrm{~s}^{-1}\right.$ for the B3 receiver) mode to cover the full velocity-width of the expected broad lines. The pointing was checked every $1-2 \mathrm{~h}$ throughout each observing run and was $\sim 3^{\prime \prime} .5$ (rms) or better. Aperture and main-beam efficiencies were derived from observations of Mars and Uranus throughout the observing runs and were compared to mean JCMT values over for the observing periods. There is excellent agreement between the two sets of values so we adopt the latter under the assumption that the mean values carry less inherent uncertainty than single measurements. The details of the observations, such as beam sizes, line frequencies, system temperatures, and total on-source integration times, are summarized in Table 1.

The HCN $1-0$ and ${ }^{13} \mathrm{CO} 2-1$ lines were observed with the Institut de Radio Astronomie Millimetrique (IRAM) $30 \mathrm{~m}$ telescope on Pico, Veleta, Spain, in 2006 June (Table 2) as

\footnotetext{
5 The JCMT is operated by the Joint Astronomy Centre on behalf of the United Kingdom Particle Physics and Astronomy Research Council (PPARC), the Netherlands Organization for Scientific Research, and the National Research Council of Canada.
}

part of a larger molecular line survey of LIRGs (Papadopoulos et al. 2007a). Each line was observed simultaneously in two polarizations using a pair of SIS receivers. The $3 \mathrm{~mm}$ A100/B100 receivers were used for the $\mathrm{HCN} 1-0$ line in conjunction with two $1 \mathrm{MHz}$ filter banks, each with 512 channels and a channel separation of $\sim 3.4 \mathrm{~km} \mathrm{~s}^{-1}$ at the observed frequency of $87 \mathrm{GHz}$. For the ${ }^{13} \mathrm{CO} 2-1$ line, we used the $1 \mathrm{~mm} \mathrm{A230/B230}$ receivers together with the two wide $4 \mathrm{MHz}$ filter banks, each with $1 \mathrm{GHz}$ bandpass and a channel separation of $\sim 5.5 \mathrm{~km} \mathrm{~s}^{-1}$ at the observed frequency of $216.5 \mathrm{GHz}$. The beam size of the $30 \mathrm{~m}$ telescope is $28^{\prime \prime}$ at $115 \mathrm{GHz}$ and $12^{\prime \prime}$ at $217 \mathrm{GHz}$. The data were acquired with the New Control System (NCS) in series of 4 minute scans, each composed of eight 30 s subscans. Total integration times were $1.2 \mathrm{~h}$ for Arp 220 and almost $2 \mathrm{~h}$ for NGC 6240 in $\mathrm{HCN} 1-0$ and ${ }^{13} \mathrm{CO} 2-1$ (Table 1). To obtain the flattest possible baselines, the wobbler switching (nutating subreflector) observing mode was employed with a frequency of $0.5 \mathrm{~Hz}$ and beam throws of $180^{\prime \prime}-240^{\prime \prime}$. Both targets were observed under good weather conditions in the late night hours with $T_{\text {sys }} \sim 95-125 \mathrm{~K}$ at $3 \mathrm{~mm}$ and $225-500 \mathrm{~K}$ at $1 \mathrm{~mm}$ on the antenna temperature $\left(T_{A}^{*}\right)$ scale. Pointing and focus were checked frequently throughout observations, and the pointing errors were found to be $\sim 3^{\prime \prime}$ (rms).

Finally, we present previously unpublished observations of Arp 220 obtained with the IRAM $30 \mathrm{~m}$ telescope during the period $1988-1992$. These data include ${ }^{13} \mathrm{CO} 1-0, \mathrm{CS} 2-$ 1 , 3-2, $\mathrm{C}^{18} \mathrm{O} 1-0$, and HNC $1-0$, the isomer of HCN. For these observations, SIS receivers were used together with the $512 \times 1 \mathrm{MHz}$ spectrometers. The observations were done with a wobbler switching of $180-240^{\prime \prime}$ in azimuth. The data were calibrated with cold and ambient loads and, depending on frequency and weather, SSB temperatures were 200-600 K. The pointing, checked with planets and quasars on a regular basis, was $\sim 3^{\prime \prime}$ (rms).

The spectra of all the molecular lines observed in Arp 220 and NGC 6240 as part of this study are shown in Figures 1 and 2 , respectively. The spectra have been plotted on the $T_{A}^{*}$ temperature scale, i.e., the antenna temperature corrected for atmospheric absorption and rearward spillover (Kutner \& Ulich 1981). The velocity-integrated line-flux densities were estimated using

$$
\begin{aligned}
I & =\int_{\Delta v} S_{v} d v=\frac{8 k_{B}}{\eta_{\mathrm{ap}} \pi D^{2}} K_{c}(x) \int_{\Delta v} T_{A}^{*} d v \\
& =\Gamma \eta_{\mathrm{ap}}^{-1} K_{c}(x) \int_{\Delta v} T_{A}^{*} d v
\end{aligned}
$$

where $\eta_{\mathrm{ap}}$ is the aperture efficiency, $D$ is the telescope diameter, and $k_{B}$ is Boltzmann's constant (Gordon et al. 1992). We find $\Gamma=15.625 \mathrm{Jy} \mathrm{K}^{-1}$ for the JCMT and $\Gamma=3.907 \mathrm{Jy} \mathrm{K}^{-1}$ for the IRAM $30 \mathrm{~m}$ telescope. $T_{A}^{*}$ is the baseline-subtracted spectrum, and $K_{c}(x)=x^{2} /\left(1-e^{-x^{2}}\right), x=\theta_{s} /\left(1.2 \theta_{\mathrm{HPBW}}\right)$ (where $\theta_{s}=$ source diameter) accounts for the coupling of the Gaussian part of the beam with a finite-sized, disk-like source. Interferometric observations have shown that the CO-emitting regions in Arp 220 and NGC 6240 are 4" in angular diameter (Tacconi et al. 1999; Scoville et al. 1997). Our line observations collect, therefore, all the flux, with minimal dependence on $K_{c}$, which is at most $\sim 1.04$ for the highest frequency (and thus smallest beam) observations reported here.

All of the measured line fluxes along with their errors are tabulated in Table 3. The errors include thermal and calibration errors, as well as errors on the assumed aperture efficiencies. 
Table 1

Observational Parameters for the Lines Observed with the JCMT

\begin{tabular}{|c|c|c|c|c|c|c|c|c|c|}
\hline$\underline{\text { Parameters }}$ & ${ }^{12} \mathrm{CO}(2-1)$ & ${ }^{12} \mathrm{CO}(3-2)$ & ${ }^{13} \mathrm{CO}(2-1)$ & ${ }^{13} \mathrm{CO}(3-2)$ & $\mathrm{HCN}(3-2)$ & $\mathrm{HCN}(4-3)$ & $\mathrm{HCO}^{+}(4-3)$ & $\mathrm{CS}(5-4)$ & $\mathrm{CS}(7-6)$ \\
\hline \multicolumn{10}{|l|}{ Arp 220} \\
\hline$v_{\text {obs }}(\mathrm{GHz})$ & 226.439 & 339.648 & 216.481 & 324.711 & 261.159 & 348.203 & 350.392 & 240.581 & 336.787 \\
\hline$T_{\text {sys }}(\mathrm{K})$ & $\sim 430$ & $350-500$ & $390-580$ & $1020-1980$ & $500-600$ & $250-360$ & $260-270$ & $280-340$ & $300-330$ \\
\hline$t_{\text {int }}(\mathrm{s})$ & 600 & 600 & 10800 & 2400 & 6000 & 5400 & 4800 & 5400 & 4200 \\
\hline \multicolumn{10}{|l|}{ NGC 6240} \\
\hline$v_{\mathrm{obs}}(\mathrm{GHz})$ & 225.025 & 337.527 & 215.128 & 322.682 & 259.528 & 346.027 & 348.203 & $\cdots$ & 334.683 \\
\hline$T_{\text {sys }}(\mathrm{K})$ & $\sim 410$ & $\sim 290$ & $400-480$ & $700-810$ & $280-290$ & $370-750$ & $310-460$ & $\cdots$ & $280-300$ \\
\hline$t_{i n t}(\mathrm{~s})$ & 600 & 600 & 13200 & 3600 & 3000 . & 7800 & 5400 & $\cdots$ & 3000 \\
\hline НРВW $\left({ }^{\prime \prime}\right)$ & 20.8 & 14.0 & 21.3 & 14.6 & 18.4 & 13.5 & 13.5 & 20.0 & 14.0 \\
\hline$\eta_{\text {ap }}$ & $0.60(0.58)^{\mathrm{a}}$ & $0.53(0.51)^{\mathrm{a}}$ & $0.60(0.58)^{\mathrm{a}}$ & $0.53(0.51)^{\mathrm{a}}$ & $0.54(0.54)^{\mathrm{a}}$ & $0.53(0.51)^{\mathrm{a}}$ & $0.53(0.51)^{\mathrm{a}}$ & $0.56(0.57)^{\mathrm{a}}$ & $0.53(0.51)^{\mathrm{a}}$ \\
\hline
\end{tabular}

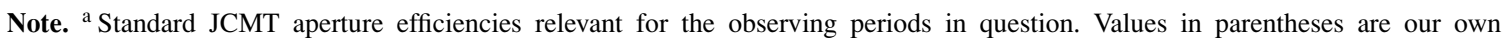
measurements.

Table 2

Observational Parameters for the Lines Observed with the IRAM 30 m Telescope

\begin{tabular}{|c|c|c|c|c|c|c|c|}
\hline Parameters & ${ }^{13} \mathrm{CO}(1-0)$ & ${ }^{13} \mathrm{CO}(2-1)$ & $\operatorname{HCN}(1-0)$ & $\mathrm{CS}(2-1)$ & $\mathrm{CS}(3-2)$ & $\mathrm{HNC}(1-0)$ & $\mathrm{C}^{18} \mathrm{O}(1-0)$ \\
\hline \multicolumn{8}{|l|}{ Arp 220} \\
\hline$v_{\mathrm{obs}}(\mathrm{GHz})$ & 108.242 & 216.481 & 87.055 & 96.238 & 144.356 & 89.051 & 107.830 \\
\hline$T_{\text {sys }}(\mathrm{K})$ & $\sim 430$ & $225-500$ & $95-125$ & $200-300$ & $200-300$ & $200-300$ & $200-300$ \\
\hline$t_{\text {int }}(\mathrm{s})$ & 600 & 4320 & 4320 & & & & \\
\hline \multicolumn{8}{|l|}{ NGC 6240} \\
\hline$v_{\mathrm{obs}}(\mathrm{GHz})$ & $\cdots$ & 215.128 & 86.512 & $\cdots$ & $\cdots$ & $\cdots$ & $\cdots$ \\
\hline$T_{\text {sys }}(\mathrm{K})$ & $\cdots$ & $225-500$ & $96-125$ & $\cdots$ & $\cdots$ & $\cdots$ & $\cdots$ \\
\hline$t_{\text {int }}(\mathrm{s})$ & $\cdots$ & 7200 & 7200 & $\ldots$ & $\ldots$ & $\ldots$ & $\ldots$ \\
\hline HPBW $(")$ & 22.4 & 11.4 & 28.3 & 25.1 & 18.0 & 27.1 & 22.4 \\
\hline$\eta_{\mathrm{ap}}$ & 0.56 & 0.41 & 0.62 & 0.59 & 0.52 & 0.59 & 0.56 \\
\hline
\end{tabular}

Each of these is assumed to be uncorrelated and thus is added in the quadrature. The latter two sources of error were found to contribute by no more than $10 \%$ each, while the thermal noise was calculated for each line using

$$
\sigma\left(\int_{\Delta v} T_{A}^{*} d v\right)=\sqrt{N_{\Delta v}}\left(1+\frac{N_{\Delta v}}{N_{\mathrm{bas}}}\right)^{1 / 2} \sigma\left(T_{A, \mathrm{ch}}^{*}\right) \Delta v_{\mathrm{ch}}
$$

where $N_{\Delta v}\left(=\Delta v / \Delta v_{\mathrm{ch}}\right)$ is the number of channels across the line, $N_{\text {bas }}$ is the number of channels (each $\Delta v_{\text {ch }}$ wide) used to estimate the baseline level, and $\sigma\left(T_{A, \text { ch }}^{*}\right)$ is the channel-tochannel rms noise, assumed to be uniform across the spectrum.

\subsection{Calibration Issues with the A3 Receiver}

At $v>230 \mathrm{GHz}$, the A3 receiver on the JCMT may present a calibration problem that has to do with the fact that the sideband ratio in this double sideband (DSB) receiver is not unity but can vary as a function of the local oscillator (LO) frequency. This may have affected our HCN 3-2 measurements, which we now reduce separately for each sideband, and intend to correct properly before coadding.

In the standard calibration, the raw normalized spectrum is scaled by $T_{\text {cal }}=\left(1+G_{\text {is }}\right) \Delta T$, where $G_{\text {is }}=G_{i} / G_{s}$ (=1 for the DSB mode) is the ratio of the image and signal sideband gains, and $\Delta T$ depends on the mean atmospheric and telescope cabin temperatures, the telescope transmission efficiency, and the lineof-sight optical depth (see Equation (A12) of Kutner \& Ulich 1981). The first step for reducing spectra for which $G_{\text {is }} \neq 1$ is to divide the spectra by 2 in order to undo the standard online calibration. Next, the lower sideband (LSB) and upper sideband (USB) tuned spectra were averaged and corrected separately using the $G_{\text {is }}\left(v_{\mathrm{LO}}\right)$ dependence given on the JCMT Web site, ${ }^{6}$ which for the HCN 3-2 line yielded $G_{\text {is }}=0.81$. Finally, the resulting corrected spectra were coadded.

\section{RESULTS}

In this section, we describe the new transitions observed in Arp 220 and NGC 6240 as part of this study, along with previously detected transitions that we have reobserved. In order to compare the line strengths not only between the two galaxies but also between different transitions within the same system, we have calculated velocity-integrated line luminosities for all of the observed transitions. These were calculated using

$$
L^{\prime}=3.25 \times 10^{7}\left(\frac{v_{\mathrm{obs}}}{\mathrm{GHz}}\right)^{-2}\left(\frac{D_{L}}{\mathrm{Mpc}}\right)^{2}(1+z)^{-3}\left(\frac{\int_{\Delta v} S_{\nu} d v}{\mathrm{Jy} \mathrm{km} \mathrm{s}^{-1}}\right),
$$

(Solomon et al. 1992b). The resulting line luminosities calculated in this manner are tabulated in Table 3.

$$
\text { 3.1. Arp } 220
$$

In Arp 220, high-resolution interferometric observations have revealed the double-horn profiles of the ${ }^{12} \mathrm{CO} 1-0$ and $2-1$ lines (Scoville et al. 1991) to originate from two nuclei embedded within a circumnuclear ring or disk (Scoville et al. 1997; Downes $\&$ Solomon 1998). We see the same line profile in our ${ }^{12} \mathrm{CO}$ 2-1 spectrum (Figure 1(a)), with a strong emission peak at $\simeq-200 \mathrm{~km} \mathrm{~s}^{-1}$ (relative to $V_{\mathrm{LSR}}=5454 \mathrm{~km} \mathrm{~s}^{-1}$ ) and a lessintense peak at $\simeq+50 \mathrm{~km} \mathrm{~s}^{-1}$. These two peaks correspond, respectively, to the western, blueshifted nucleus and to the

\footnotetext{
6 http://www.jach.hawaii.edu/JCMT/spectral_line/Instrument_homes/RxA3i/
} sidebands.html. 


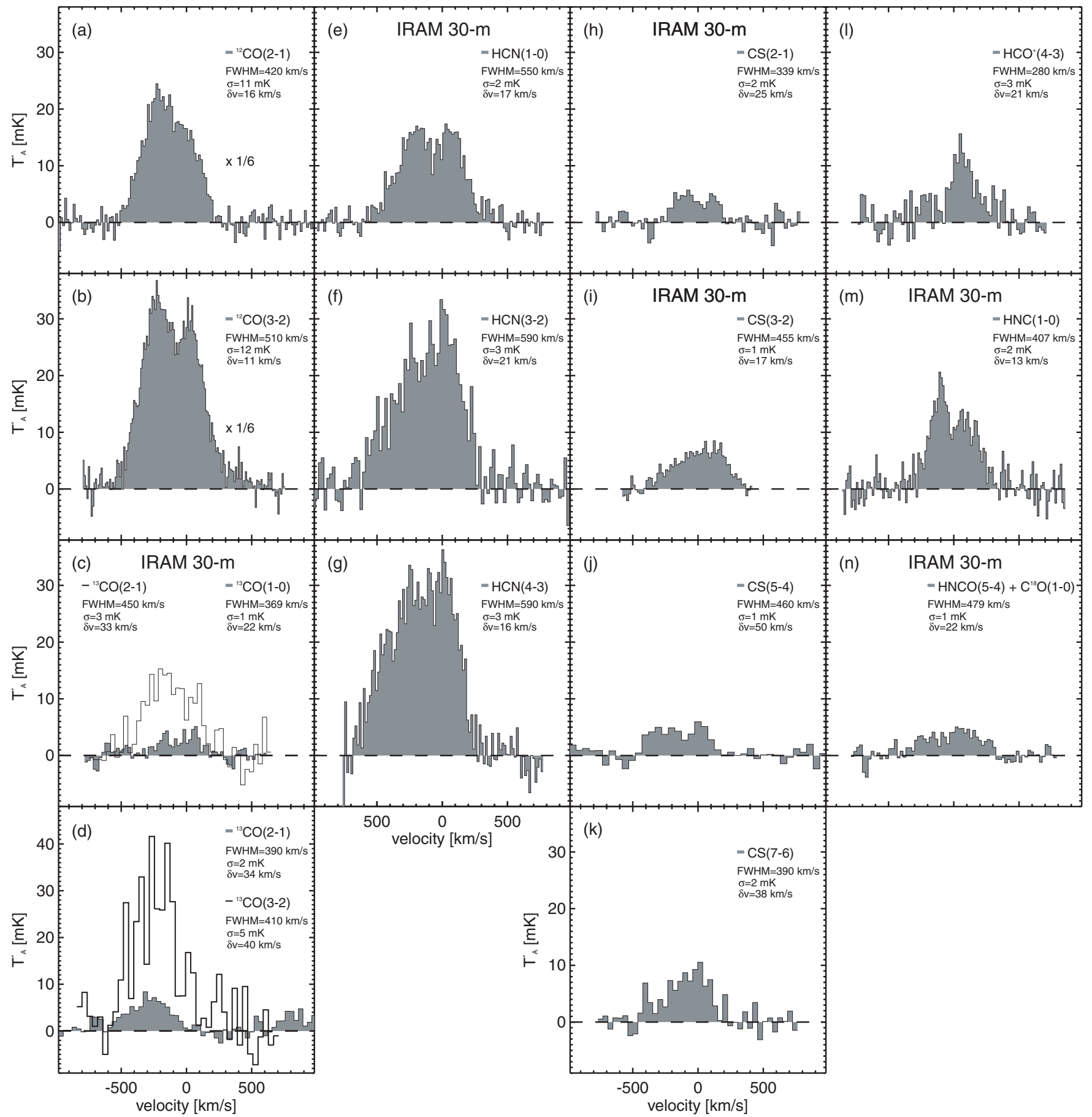

Figure 1. Molecular line spectra observed in Arp 220. The ${ }^{12} \mathrm{CO} 2-1$ and 3-2 spectra have been scaled down by a factor of 6 in order to fit on the plot. The velocity-axis is relative to $v_{\mathrm{LSR}}=5454 \mathrm{~km} \mathrm{~s}^{-1}$. The FWHM line width and channel-to-channel rms noise are given for each line. All spectra were obtained with the JCMT except for those in panel (c), (e), (h)-(i), and (m)-(n) which are from the IRAM $30 \mathrm{~m}$ telescope.

eastern, redshifted nucleus in the molecular disk (Downes \& Solomon 1998). Our ${ }^{12}$ CO 3-2 line, single-dish spectrum shows a double-horn profile similar to the lower transitions (Lisenfeld et al. 1996; Mauersberger et al. 1999).

One subtle difference exists however, and is confirmed by our own ${ }^{12} \mathrm{CO} 3-2$ spectrum (Figure 1(b)). The relative strength of the peak at $\simeq+50 \mathrm{~km} \mathrm{~s}^{-1}$ is greater in the 3-2 line than in the $1-0$ and $2-1$ spectra. In the 3-2 spectrum, the western component is only slightly stronger than the eastern. Recent interferometric observations with the Submillimeter Array (SMA) showed that the CO 3-2 and the $890-\mu \mathrm{m}$ dust emission is indeed strongest in the western nucleus (Sakamoto et al. 2008).

The ${ }^{13} \mathrm{CO}$ lines observed in Arp 220 with the IRAM $30 \mathrm{~m}$ telescope and the JCMT are shown in Figures 1(c) and (d), respectively. Although the ${ }^{13} \mathrm{CO}$ lines seem somewhat narrower than their ${ }^{12} \mathrm{CO}$ counterparts, this may be simply an effect of the low signal-to-noise ratio $(\mathrm{S} / \mathrm{N})$ in the line wings and the uncertain baseline level. In general, all the ${ }^{12} \mathrm{CO}$ and ${ }^{13} \mathrm{CO}$ lines seem to have very similar line profiles, within the available $\mathrm{S} / \mathrm{N}$. Both the ${ }^{13} \mathrm{CO} 1-0$ and 2-1 spectra observed with the IRAM $30 \mathrm{~m}$ telescope show tentative evidence of double peaks, 


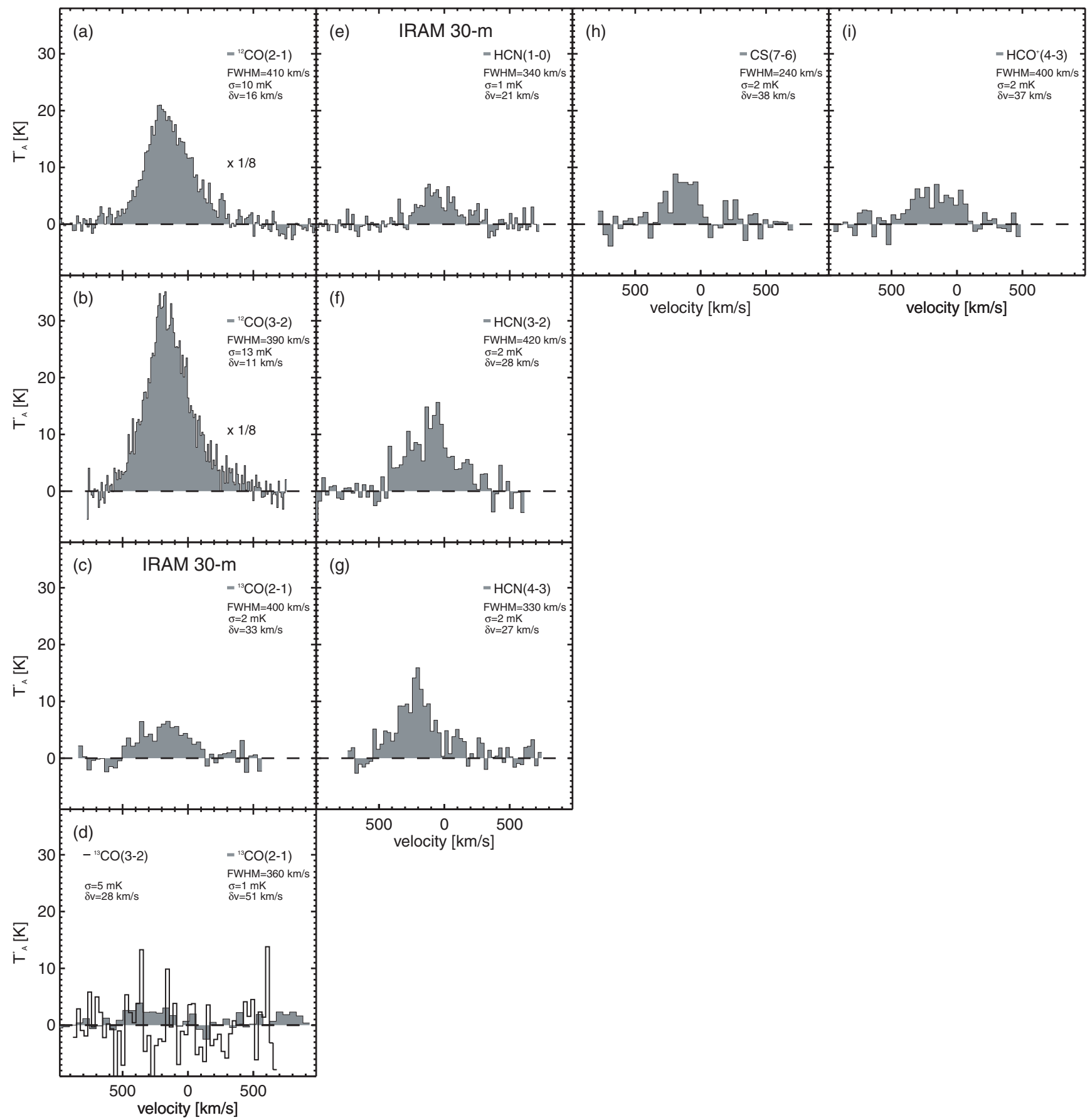

Figure 2. Molecular line spectra observed in NGC 6240 . The velocity axis is relative to $v_{\mathrm{LSR}}=7359 \mathrm{~km} \mathrm{~s}^{-1}$, and the ${ }^{12} \mathrm{CO} 2-1$ and $3-2$ spectra have been scaled down by a factor of 8. All spectra were obtained with the JCMT except for those in panel (c) and (e) which are from the IRAM $30 \mathrm{~m}$ telescope. Other details are the same as in Figure 1.

consistent with the ${ }^{12} \mathrm{CO}$ lines. In the $1-0$ spectrum, both peaks are equally strong, whereas the peak at $\simeq-200 \mathrm{~km} \mathrm{~s}^{-1}$ is stronger in the $2-1$ spectrum. This also appears to be the case in the 2-1 and 3-2 spectra observed with the JCMT (Figure 1(d)), although we note the 3-2 spectrum in particular is very noisy. The fact that the ${ }^{13} \mathrm{CO} 2-1$ spectra obtained with the two telescopes are in agreement in terms of line shape as well as flux density gives us confidence that our observations are able to robustly discern the line shapes of even faint lines such as ${ }^{13} \mathrm{CO} 2-1$.

The HCN 1-0, 3-2, and 4-3 spectra observed in Arp 220 are shown in Figures 1(e)-(g), respectively. The HCN 1-0 spectrum shows a clear double-horn profile similar to ${ }^{12} \mathrm{CO} 3-2$, with equally strong peaks. The $\mathrm{HCN} 3-2$ and 4-3 line profiles are both very broad $\left(\mathrm{FWHM} \simeq 540-590 \mathrm{~km} \mathrm{~s}^{-1}\right)$ and share the same overall shape. They both show evidence of double peaks with the $\simeq+50 \mathrm{~km} \mathrm{~s}^{-1}$ component being more prominent. The shape is unlike the $\mathrm{HCN}(1-0)$ spectrum - as well as the ${ }^{12} \mathrm{CO}$ $2-1$ and $3-2$ spectra-where the $\simeq-200 \mathrm{~km} \mathrm{~s}^{-1}$ component is brighter.

The CS lines-of which only CS 3-2 has been previously reported for Arp 220-trace a much overlapping excitation range with that of $\mathrm{HCN}$ (see Table 6), and are shown in Figures 1(h)-(k). All CS lines have double-horn profiles, with 
Table 3

Molecular and Atomic Transitions Detected Toward Arp 220 and NGC 6240

\begin{tabular}{|c|c|c|c|c|c|c|c|c|c|c|}
\hline \multirow[t]{2}{*}{ Line } & \multicolumn{2}{|c|}{ Arp 220} & \multirow[t]{2}{*}{$L^{\prime c}$} & \multirow[t]{2}{*}{ Telescope } & \multirow[t]{2}{*}{ References } & \multirow[t]{2}{*}{ FWHM $^{\mathrm{a}}$} & \multicolumn{2}{|c|}{ NGC 6240} & \multirow[t]{2}{*}{ Telescope } & \multirow[t]{2}{*}{ References } \\
\hline & FWHM $^{\mathrm{a}}$ & $S_{v} \Delta v^{\mathrm{b}}$ & & & & & $S_{v} \Delta v^{\mathrm{b}}$ & $L^{\prime \mathrm{c}}$ & & \\
\hline \multirow[t]{4}{*}{${ }^{12} \mathrm{CO}(1-0)$} & 504 & $410 \pm 41$ & $5.9 \pm 0.6$ & OVRO & [1] & 370 & $314 \pm 64$ & $8.3 \pm 1.7$ & IRAM $30 \mathrm{~m}$ & [3] \\
\hline & $\ldots$ & $384 \pm 115^{\mathrm{d}}$ & $5.5 \pm 1.7$ & OVRO & {$[2]$} & 469 & $324 \pm 33$ & $8.6 \pm 0.9$ & OVRO & [18] \\
\hline & 480 & $496 \pm 99$ & $7.2 \pm 1.4$ & IRAM $30 \mathrm{~m}$ & {$[3]$} & & $322 \pm 29$ & $8.5 \pm 0.8$ & & \\
\hline & & $419 \pm 36$ & $6.0 \pm 0.5$ & & & & & & & \\
\hline \multirow[t]{7}{*}{${ }^{12} \mathrm{CO}(2-1)$} & 447 & $1071 \pm 107$ & $3.9 \pm 0.4$ & OVRO & {$[1]$} & 300 & $1220 \pm 366^{\mathrm{d}}$ & $8.1 \pm 2.4$ & IRAM PdBI & [19] \\
\hline & $\ldots$ & $1088 \pm 330^{\mathrm{d}}$ & $3.9 \pm 1.2$ & IRAM $30 \mathrm{~m}$ & {$[4]$} & 410 & $1741 \pm 350$ & $11.6 \pm 2.3$ & JCMT & This work \\
\hline & $\ldots$ & $1118 \pm 112$ & $4.0 \pm 0.4$ & NRAO $12 \mathrm{~m}$ & [5] & & $1492 \pm 253$ & $9.9 \pm 1.7$ & & \\
\hline & 380 & $1100 \pm 220$ & $4.0 \pm 0.8$ & OVRO & [6] & & & & & \\
\hline & & $1730 \pm 519$ & $6.2 \pm 1.9$ & JCMT & [9] & & & & & \\
\hline & 420 & $1549 \pm 311$ & $5.6 \pm 1.1$ & JCMT & This work & & & & & \\
\hline & & $1127 \pm 69$ & $4.1 \pm 0.3$ & & & & & & & \\
\hline \multirow[t]{5}{*}{${ }^{12} \mathrm{CO}(3-2)$} & $\ldots$ & $4094 \pm 758$ & $6.6 \pm 1.2$ & JCMT & [7] & 390 & $3205 \pm 642$ & $9.5 \pm 1.9$ & JCMT & This work \\
\hline & 306 & $4154 \pm 975$ & $6.7 \pm 1.6$ & HHT & {$[8]$} & & & & & \\
\hline & & $3700 \pm 1110^{d}$ & $5.9 \pm 1.8$ & JCMT & [9] & & & & & \\
\hline & 510 & $3168 \pm 634$ & $5.1 \pm 1.0$ & JCMT & This work & & & & & \\
\hline & & $3674 \pm 405$ & $5.9 \pm 0.6$ & & & & & & & \\
\hline \multirow[t]{2}{*}{${ }^{13} \mathrm{CO}(1-0)$} & $\ldots$ & $\leqslant 9.4$ & $\leqslant 0.15$ & SEST & [5] & $\ldots$ & $6.5 \pm 1.9$ & $0.19 \pm 0.06$ & IRAM $30 \mathrm{~m}$ & {$[20]$} \\
\hline & 369 & $9 \pm 2$ & $0.14 \pm 0.03$ & IRAM $30 \mathrm{~m}$ & This work & & & & & \\
\hline \multirow[t]{5}{*}{${ }^{13} \mathrm{CO}(2-1)$} & $\ldots$ & $56 \pm 17^{\mathrm{d}}$ & $0.22 \pm 0.07$ & IRAM $30 \mathrm{~m}$ & [10] & 360 & $28 \pm 7$ & $0.20 \pm 0.05$ & JCMT & This work \\
\hline & $\ldots$ & $54 \pm 11$ & $0.21 \pm 0.04$ & JCMT & {$[11]$} & 400 & $25 \pm 5$ & $0.18 \pm 0.04$ & IRAM $30 \mathrm{~m}$ & This work \\
\hline & 450 & $60 \pm 13$ & $0.24 \pm 0.05$ & IRAM $30 \mathrm{~m}$ & This work & & $26 \pm 4$ & $0.19 \pm 0.03$ & & \\
\hline & 390 & $70 \pm 16$ & $0.28 \pm 0.06$ & JCMT & This work & & & & & \\
\hline & & $59 \pm 7$ & $0.23 \pm 0.03$ & & & & & & & \\
\hline${ }^{13} \mathrm{CO}(3-2)$ & 410 & $404 \pm 86$ & $0.71 \pm 0.15$ & JCMT & This work & $\ldots$ & $\leqslant 79$ & $\leqslant 0.3$ & JCMT & This work \\
\hline${ }^{12} \mathrm{C}^{18} \mathrm{O}(1-0)$ & 280 & $9 \pm 2$ & $0.14 \pm 0.03$ & IRAM $30 \mathrm{~m}$ & This work & $\ldots$ & $\ldots$ & $\ldots$ & $\ldots$ & $\ldots$ \\
\hline $\mathrm{HNCO}(5-4)$ & 280 & $5 \pm 2$ & $0.08 \pm 0.03$ & IRAM $30 \mathrm{~m}$ & This work & $\ldots$ & $\ldots$ & $\ldots$ & $\ldots$ & $\ldots$ \\
\hline \multirow[t]{5}{*}{$\mathrm{HCN}(1-0)$} & $\ldots$ & $35 \pm 11^{\mathrm{d}}$ & $0.85 \pm 0.27$ & IRAM PdBI & [12] & $\ldots$ & $26 \pm 8^{\mathrm{d}}$ & $1.17 \pm 0.36$ & IRAM $30 \mathrm{~m}$ & [13] \\
\hline & 550 & $59 \pm 12$ & $1.44 \pm 0.29$ & IRAM $30 \mathrm{~m}$ & This work & $\ldots$ & $14 \pm 4^{\mathrm{d}}$ & $0.63 \pm 0.18$ & NMA & [21] \\
\hline & $\ldots$ & $38 \pm 12$ & $0.93 \pm 0.29$ & IRAM $30 \mathrm{~m}$ & [13] & 340 & $13 \pm 3$ & $0.58 \pm 0.13$ & IRAM $30 \mathrm{~m}$ & This work \\
\hline & 530 & $48 \pm 14$ & $1.17 \pm 0.34$ & IRAM $30 \mathrm{~m}$ & {$[22]$} & 410 & $16 \pm 5$ & $0.72 \pm 0.22$ & IRAM $30 \mathrm{~m}$ & [22] \\
\hline & & $44 \pm 6$ & $1.08 \pm 0.15$ & & & & $15 \pm 2$ & $0.66 \pm 0.09$ & & \\
\hline $\operatorname{HCN}(2-1)$ & 530 & $144 \pm 43$ & $0.88 \pm 0.26$ & IRAM $30 \mathrm{~m}$ & [22] & 410 & $45 \pm 13$ & $0.51 \pm 0.15$ & IRAM $30 \mathrm{~m}$ & [22] \\
\hline \multirow[t]{3}{*}{$\mathrm{HCN}(3-2)$} & 540 & $361 \pm 73$ & $0.98 \pm 0.20$ & JCMT & This work & 430 & $160 \pm 33$ & $0.80 \pm 0.16$ & JCMT & This work \\
\hline & 530 & $258 \pm 77$ & $0.70 \pm 0.21$ & IRAM $30 \mathrm{~m}$ & [22] & 410 & $74 \pm 22$ & $0.37 \pm 0.11$ & IRAM $30 \mathrm{~m}$ & [22] \\
\hline & & $312 \pm 53$ & $0.85 \pm 0.14$ & & & & $100 \pm 18$ & $0.51 \pm 0.09$ & & \\
\hline $\mathrm{HCN}(4-3)$ & 590 & $587 \pm 118$ & $0.90 \pm 0.18$ & JCMT & This work & 320 & $138 \pm 29$ & $0.39 \pm 0.08$ & JCMT & This work \\
\hline $\mathrm{HNC}(1-0)$ & $516 \pm 38$ & $55 \pm 11$ & $1.28 \pm 0.26$ & IRAM $30 \mathrm{~m}$ & [14] & $\ldots$ & $14 \pm 7$ & $0.60 \pm 0.30$ & SEST & [15] \\
\hline & .. & $34 \pm 7$ & $0.79 \pm 0.16$ & SEST & [15] & & & & & \\
\hline & 407 & $43 \pm 8$ & $0.96 \pm 0.19$ & IRAM $30 \mathrm{~m}$ & This work & & & & & \\
\hline & & $40 \pm 5$ & $0.94 \pm 0.11$ & & & & & & & \\
\hline $\mathrm{CS}(2-1)$ & 339 & $12 \pm 3$ & $0.24 \pm 0.06$ & IRAM $30 \mathrm{~m}$ & This work & $\ldots$ & $\ldots$ & $\ldots$ & $\ldots$ & $\ldots$ \\
\hline $\operatorname{CS}(3-2)$ & $\ldots$ & $22 \pm 7^{\mathrm{d}}$ & $0.20 \pm 0.06$ & IRAM $30 \mathrm{~m}$ & [10] & $\ldots$ & $\ldots$ & $\ldots$ & $\ldots$ & $\ldots$ \\
\hline & 455 & $26 \pm 5$ & $0.24 \pm 0.04$ & IRAM $30 \mathrm{~m}$ & This work & & & & & \\
\hline & & $25 \pm 4$ & $0.23 \pm 0.03$ & & & & & & & \\
\hline $\mathrm{CS}(5-4)$ & 460 & $68 \pm 15$ & $0.22 \pm 0.05$ & JCMT & This work & $\ldots$ & $\ldots$ & $\ldots$ & $\ldots$ & $\ldots$ \\
\hline $\operatorname{CS}(7-6)$ & 390 & $111 \pm 24$ & $0.18 \pm 0.04$ & JCMT & This work & 240 & $60 \pm 14$ & $0.18 \pm 0.04$ & JCMT & This work \\
\hline $\mathrm{HCO}^{+}(1-0)$ & $\ldots$ & $20 \pm 6^{\mathrm{d}}$ & $0.48 \pm 0.14$ & IRAM PdBI & [12] & 420 & $21 \pm 3$ & $0.93 \pm 0.13$ & NMA & [21] \\
\hline & $\ldots$ & $19 \pm 6$ & $0.46 \pm 0.14$ & IRAM $30 \mathrm{~m}$ & {$[13]$} & $\ldots$ & $25 \pm 8^{\mathrm{d}, \mathrm{e}}$ & $1.11 \pm 0.35$ & IRAM $30 \mathrm{~m}$ & {$[16]$} \\
\hline & $\ldots$ & $22 \pm 7^{\mathrm{d}, \mathrm{e}}$ & $0.53 \pm 0.17$ & IRAM $30 \mathrm{~m}$ & [16] & & $21 \pm 3$ & $0.95 \pm 0.12$ & & \\
\hline & & $20 \pm 4$ & $0.49 \pm 0.09$ & & & & & & & \\
\hline $\mathrm{HCO}^{+}(3-2)$ & $\ldots$ & $49 \pm 15^{\mathrm{d}, \mathrm{e}}$ & $0.13 \pm 0.04$ & IRAM $30 \mathrm{~m}$ & [16] & $\ldots$ & $47 \pm 14^{\mathrm{d}, \mathrm{e}}$ & $0.23 \pm 0.07$ & IRAM $30 \mathrm{~m}$ & [16] \\
\hline $\mathrm{HCO}^{+}(4-3)$ & 280 & $106 \pm 23$ & $0.16 \pm 0.03$ & JCMT & This work & 400 & $71 \pm 17$ & $0.20 \pm 0.05$ & JCMT & This work \\
\hline $\mathrm{C} \mathrm{I}\left({ }^{3} P_{1}-{ }^{3} P_{0}\right)$ & & $1160 \pm 350$ & & JCMT & [17] & & $600 \pm 180$ & & JCMT & [17] \\
\hline
\end{tabular}

Notes.

${ }^{a}$ Line widths are in $\mathrm{km} \mathrm{s}^{-1}$.

${ }^{\mathrm{b}}$ Line flux densities are in units of $\mathrm{Jy} \mathrm{km} \mathrm{s}^{-1}$.

${ }^{\mathrm{c}}$ Line luminosities are in units of $\times 10^{9} \mathrm{~K} \mathrm{~km} \mathrm{~s}^{-1} \mathrm{pc}^{2}$.

${ }^{\mathrm{d}}$ We assume a $30 \%$ error on the flux measurement.

e The fluxes are derived from the luminosities quoted by Graciá-Carpio et al. (2006).

References. (1) Scoville et al. (1997); (2) Scoville et al. (1991); (3) Solomon et al. (1997); (4) Radford et al. (1991a); (5) Aalto et al. (1995); (6) Downes \& Solomon (1998); (7) Lisenfeld et al. (1996); (8) Mauersberger et al. (1999); (9) Wiedner et al. (2002); (10) Solomon et al. (1990); (11) Papadopoulos \& Seaquist (1998); (12) Radford et al. (1991b); (13) Solomon et al. (1992a); (14) Hüttemeister et al. (1995); (15) Aalto et al. (2002); (16) Graciá-Carpio et al. (2006); (17) Papadopoulos \& Greve (2004); (18) Bryant \& Scoville (1999); (19) Tacconi et al. (1999); (20) Casoli et al. (1992); (21) Nakanishi et al. (2005); (22) Krips et al. (2008). 
the $\simeq+50 \mathrm{~km} \mathrm{~s}^{-1}$ peak stronger in the CS 3-2, 5-4, and 7-6 spectra, while the two peaks are almost the same strength in the $2-1$ spectrum. This mimics the observed evolution from low- to high- $J$ in the HCN line profiles. We note, however, the apparent asymmetry of the CS 3-2 line may be an artifact of the limited receiver bandwidth. In determining the baseline, we forced the right edge of this spectrum to zero, so our line-flux estimate for this line is likely an underestimate.

Finally, in the last column of Figure 1, we show the $\mathrm{HCO}^{+}$ $4-3$, HNC $1-0$, and the blended $\mathrm{C}^{18} \mathrm{O} 1-0$ and HNCO $5_{0,5}-4_{0,4}$ lines. Similarly to the $\mathrm{HCN}$ and $\mathrm{CS}$ lines, the $\mathrm{HCO}^{+}$spectrum peaks at $\simeq+50 \mathrm{~km} \mathrm{~s}^{-1}$, and shows some faint emission at $\simeq-200 \mathrm{~km} \mathrm{~s}^{-1}$, which corresponds to the stronger ${ }^{12} \mathrm{CO}$ feature. In this respect, the $\mathrm{HCO}^{+}$spectrum looks similar to CS 7-6, where the western component is almost completely quenched. In stark contrast to this, the double-horn spectrum of HNC 1-0 shows a remarkably strong emission peak at $\simeq-200 \mathrm{~km} \mathrm{~s}^{-1}$, suggesting a substantial fraction of the HNC is concentrated in the western nucleus. The blended $\mathrm{C}^{18} \mathrm{O} 1-$ 0 and HNCO $5_{0,5}-4_{0,4}$ spectrum is shown in Figure 1(n). In order to determine separate parameters for $\mathrm{C}^{18} \mathrm{O} 1-0$ and $\mathrm{HNCO}$ $5_{0,5}-4_{0,4}$, two Gaussian components with the same width and fixed separation were fitted. From the resulting intensities we calculated integrated line fluxes and luminosities (Table 3).

It means that the double-horn structure seen in the low- $J$ ${ }^{12} \mathrm{CO}$ spectra is also discernible in the high- $J$ spectra of $\mathrm{HCN}$, $\mathrm{CS}$, and $\mathrm{HCO}^{+}$, albeit with the relative strengths of the two peaks reversed. In fact, a rather clear trend is apparent: the $\simeq-200 \mathrm{~km} \mathrm{~s}^{-1}$ peak is stronger in the ${ }^{12} \mathrm{CO} 2-1$ spectrum, the peaks are about equally strong in the ${ }^{12} \mathrm{CO} 3-2$ and HCN $1-0$ profiles, and the $\simeq+50 \mathrm{~km} \mathrm{~s}^{-1}$ peak is stronger in the HCN 3-2 and 4-3 spectra. Thus, as we climb the $J$-ladder and probe higher and higher critical densities, the strength of the emission shifts from the western to the eastern nucleus. This picture is confirmed by the CS 7-6, and $\mathrm{HCO}^{+} 4-3$ spectra which have even higher critical densities than the HCN $1-0$ line and are completely dominated by the $\simeq+50 \mathrm{~km} \mathrm{~s}^{-1}$ component with very little or no emission at all at $\simeq-200 \mathrm{~km} \mathrm{~s}^{-1}$.

An important point to make here is that it is only due to the high-density gas tracers such as $\mathrm{HCO}^{+}, \mathrm{HCN}$, and $\mathrm{CS}$ that we are able to conclude that the eastern nucleus harbors most of the dense gas. Without those, we would have been inclined to conclude, based on the CO 3-2 spectrum (Figure 1(b)) and its distribution (Sakamoto et al. 2008), that most of the dense gas was contained in the western component.

Given the modest (yet real) differences of the relative line strengths across the velocity range of Arp 220, and for reasons of simplicity and straightforward comparison to NGC 6240, we decided to use the line intensities average over its entire profile and the associated line ratios in our subsequent analysis (Section 4).

\subsection{NGC 6240}

The ${ }^{12} \mathrm{CO} 2-1$ and 3-2 spectra of NGC 6240 are shown in Figures 2(a) and (b), respectively. Unlike Arp 220, the ${ }^{12} \mathrm{CO}$ 3-2 and 2-1 spectra in NGC 6240 have almost identical shapes, with spectra having a single-peaked profile, skewed toward the blue $\left(\simeq-200 \mathrm{~km} \mathrm{~s}^{-1}\right.$ relative to $\left.v_{\mathrm{LSR}}=7359 \mathrm{~km} \mathrm{~s}^{-1}\right)$. This is consistent with high-resolution interferometric observations of low- $J{ }^{12} \mathrm{CO}$ lines in the inner regions of NGC 6240, which suggest more than half of the molecular gas resides in a central structure dominated by turbulent flows, rather than systematic orbital motion (Tacconi et al. 1999). The ${ }^{13} \mathrm{CO}$
2-1 spectra obtained with the JCMT and IRAM $30 \mathrm{~m}$ are shown in Figures 2(c) and (d), respectively, and both appear similar in shape to their ${ }^{12} \mathrm{CO}$ counterparts. Unfortunately, we cannot compare the ${ }^{13} \mathrm{CO} 3-2$ spectrum (Figure 2(d)) as no obvious detection of this transition was made. We are able, however, to set a sensitive upper limit on its integrated flux (Table 3).

The HCN 1-0, 3-2, and 4-3 transitions observed in NGC 6240 are shown in Figures 2(e)-(g). Although the HCN 1-0 line has previously been detected (Solomon et al. 1992a; Tacconi et al. 1999; Nakanishi et al. 2005), this is the first time the 3-2 and 4-3 transitions have been detected in this galaxy. All three $\mathrm{HCN}$ lines peak at $\simeq-200 \mathrm{~km} \mathrm{~s}^{-1}$ and are consistent with the CO line profiles.

Unlike Arp 220, where several CS lines were observed, only the 7-6 line was observed in NGC 6240 due to limited observing time. The spectrum is shown in Figure 2(h). This is the only detection of CS in NGC 6240 to date and the line profile is consistent with those of $\mathrm{CO}$ and $\mathrm{HCN}$.

Finally, the $\mathrm{HCO}^{+} 4-3$ spectrum observed in NGC 6240 is shown in Figure 2(i). The $\mathrm{HCO}^{+} 4-3$ line profile is in good agreement with the 1-0 line (Nakanishi et al. 2005) and is consistent with a single peak at $\simeq-200 \mathrm{~km} \mathrm{~s}^{-1}$.

In summary, all of the transitions detected in NGC 6240 exhibit a similar, Gaussian-like velocity profile, peaking at about the same velocity $\left(\simeq-200 \mathrm{~km} \mathrm{~s}^{-1}\right)$, in contrast to Arp 220 where all the lines not only show a double-peaked profile but a strong evolution in the dominant peak is also discernible. The similar velocity profile over such a large range of densities probed in NGC 6240 signifies a kinematical state of the molecular gas where the various phases remain well mixed down to much smaller scales than in Arp 220 where the denser phases seem settled to two counter-rotating dense gas disks, enveloped by a much more diffuse CO-bright gas phase. Subarcsec interferometric imaging of the HCN, CS, and $\mathrm{HCO}^{+}$imaging of these systems is valuable in confirming such a picture, a possible result of the different merger state of these two strongly interacting systems.

\subsection{Literature Data}

A major goal of this study was to use our observations as well as results from the literature to compile a large, identical molecular line catalog for Arp 220 and NGC 6240. Thus the literature was scanned for all relevant molecular line measurements and for each transition the weighted average of every independent flux measurement was calculated. In cases where a measurement deviated significantly from the mean for obvious reasons (limited bandwidth, poor conditions, etc.), it was discarded from the average. Note that often in the literature line measurements are given in (main beam or antenna) temperature units and only rarely in Janskys. In those cases, we used the aperture and/or main-beam efficiencies quoted in the paper or, if those were not given, the average values for the particular telescope (typically tabulated in the observing manual). The final tally of lines is given in Table 3 along with their corresponding fluxes and global line luminosities. Global line ratios were calculated as the velocity/area averaged brightness temperature ratios (equivalent to taking the ratio of the line luminosities) and are listed in Tables 4 and 5.

Our observations of ${ }^{12} \mathrm{CO} 2-1$ in both Arp 220 and NGC 6240 yield somewhat higher line fluxes than previous single-dish and interferometry observations listed in Table 3, although, we note that Wiedner et al. (2002) using the JCMT measured an even 
Table 4

CO Line Ratios for Arp 220 and NGC 6240

\begin{tabular}{|c|c|c|c|c|c|c|}
\hline \multirow[t]{3}{*}{ Galaxy } & ${ }^{12} \mathrm{CO}(2-1)$ & ${ }^{12} \mathrm{CO}(3-2)$ & ${ }^{12} \mathrm{CO}(1-0)$ & ${ }^{12} \mathrm{CO}(2-1)$ & ${ }^{12} \mathrm{CO}(3-2)$ & $\mathrm{C}^{18} \mathrm{O}(1-0)$ \\
\hline & $\overline{{ }^{12} \mathrm{CO}(1-0)}$ & $\overline{{ }^{12} \mathrm{CO}(1-0)}$ & $\overline{{ }^{13} \mathrm{CO}(1-0)}$ & $\overline{{ }^{13} \mathrm{CO}(2-1)}$ & $\overline{{ }^{13} \mathrm{CO}(3-2)}$ & $\overline{{ }^{13} \mathrm{CO}(1-0)}$ \\
\hline & $r_{21}$ & $r_{32}$ & $\mathcal{R}_{10}$ & $\mathcal{R}_{21}$ & $\mathcal{R}_{32}$ & $\mathcal{R}_{10}^{(18)}$ \\
\hline Arp 220 & $0.7 \pm 0.1$ & $1.0 \pm 0.1$ & $43 \pm 10$ & $18 \pm 3$ & $8 \pm 2$ & $1.0 \pm 0.3$ \\
\hline NGC 6240 & $1.2 \pm 0.2$ & $1.1 \pm 0.2$ & $45 \pm 15$ & $52 \pm 12$ & $\geqslant 32$ & $\ldots$ \\
\hline
\end{tabular}

Table 5

Observed and Modeled HCN, CS and $\mathrm{HCO}^{+}$Line Ratios for Arp 220 and NGC 6240

\begin{tabular}{|c|c|c|c|c|c|c|c|c|c|}
\hline \multirow[t]{2}{*}{ Galaxy } & $\mathrm{HCN}(2-1)$ & $\mathrm{HCN}(3-2)$ & $\mathrm{HCN}(4-3)$ & $\mathrm{HCN}(1-0)$ & $\mathrm{CS}(3-2)$ & $\mathrm{CS}(5-4)$ & $\operatorname{CS}(7-6)$ & $\mathrm{HCO}^{+}(3-2)$ & $\mathrm{HCO}^{+}(4-3)$ \\
\hline & $\mathrm{HCN}(1-0)$ & $\mathrm{HCN}(1-0)$ & $\mathrm{HCN}(1-0)$ & $\mathrm{HNC}(1-0)$ & $\mathrm{CS}(2-1)$ & $\mathrm{CS}(2-1)$ & $\mathrm{CS}(2-1)$ & $\mathrm{HCO}^{+}(1-0)$ & $\mathrm{HCO}^{+}(1-0)$ \\
\hline \multicolumn{10}{|l|}{ Arp 220} \\
\hline Observed & $0.8 \pm 0.3$ & $0.8 \pm 0.2$ & $0.8 \pm 0.2$ & $1.2 \pm 0.2$ & $1.0 \pm 0.3$ & $1.0 \pm 0.3$ & $0.8 \pm 0.3$ & $0.27 \pm 0.10$ & $0.33 \pm 0.09$ \\
\hline $\begin{array}{l}\text { Modeled } \\
\text { NGC } 6240\end{array}$ & 1.0 & 0.9 & 0.8 & $\ldots$ & 1.0 & $0.9-1.0$ & $0.8-0.9$ & $0.3-0.4$ & 0.4 \\
\hline Observed & $0.8 \pm 0.3$ & $0.8 \pm 0.2$ & $0.6 \pm 0.1$ & $1.1 \pm 0.6$ & $\ldots$ & $\ldots$ & $\ldots$ & $0.24 \pm 0.08$ & $0.21 \pm 0.06$ \\
\hline Modeled & $0.9-1.0$ & $0.8-0.9$ & $0.6-0.7$ & $\ldots$ & $\ldots$ & $\ldots$ & $\cdots$ & $0.3-0.4$ & 0.4 \\
\hline
\end{tabular}

larger line flux in Arp 220 than ours. A careful scrutiny of the individual subscans in our data and of the calibration on the dates the observations were made revealed nothing out of the ordinary. In fact, given that each source was observed on different nights, yet both have $\sim 40 \%$ higher line fluxes than previously published values, suggest that the offset may well be real. A close inspection of previously published ${ }^{12} \mathrm{CO} 2-1$ spectra reveals they all suffer from either limited bandwidth (the case for single-dish measurements), and/or the sources have been overresolved spatially (in the case of interferometric observations). Because only few observations favor the higher ${ }^{12} \mathrm{CO} 2-1$ fluxes in Arp 220 and NGC 6240, however, we conservatively adopt the mean flux value of all available measurements for our analysis. The HCN 4-3 line in Arp 220 was first observed by Wiedner et al. (2002), who found a line flux only half as large as ours. Here, we adopt our own measurement since our spectrum has a higher $\mathrm{S} / \mathrm{N}$ than that of Wiedner et al. (2002), and the latter used aperture efficiencies which deviate significantly from the standard values given for JCMT.

In NGC 6240 we find a HCN 1-0 flux that is half the previously published single-dish measurement (Solomon et al. 1992a) but is in excellent agreement with the interferometric measurement by Nakanishi et al. (2005). Because our data were obtained over the course of 2 days in excellent weather conditions and because a careful reduction of the data from each day separately yielded consistent results, we find the lower values more likely. In the case of Arp 220, our estimate of the $\mathrm{HCN} 1-0$ line flux is about $35 \%$ larger than that of Solomon et al. (1992a), which is just within the errors. Recent, independent observations of the HCN 1-0 line in Arp 220 and NGC 6240 with the IRAM $30 \mathrm{~m}$ telescope yield line fluxes in agreement with our measurements (J. Graciá-Carpio 2006, private communication), suggesting the HCN 1-0 line fluxes given by Solomon et al. (1992a) may have been affected by systematic calibration uncertainties. Nonetheless, for the line ratio analysis in this paper, we conservatively adopt the weighted average of all three HCN 1-0 measurements in these two systems.

We end this section by noting that Table 3 constitutes the most extensive molecular line catalog available for any (U)LIRG. In terms of extragalactic objects in general, it is matched only by data published for the much less extreme starburst galaxies M 82 and NGC 253 (e.g., Weiß et al. 2005; Seaquist et al. 2006;
Bayet et al. 2004; Bradford et al. 2003). However, unlike M 82 and NGC 253, where only the ${ }^{12} \mathrm{CO} 1-0$ and $2-1$ transitions have been observed over their entire extent (e.g., Weiß et al. 2005), our study picks up the global emission from Arp 220 and NGC 6240 for all transitions, allowing for an unbiased study of the properties of their entire molecular gas reservoir using the corresponding millimeter/submillimeter line ratios. This mirrors observations of the millimeter/submillimeter lines observed in high- $z$ starbursts where much fewer lines are available, and thus cannot yield meaningful constraints on the conditions or the mass per gas phase for their molecular gas reservoirs.

\section{ANALYSIS}

The first indication that the molecular ISM in extreme starburst galaxies is fundamentally different from that of normal galaxies came from the observation that the former have much larger ${ }^{12} \mathrm{CO} /{ }^{13} \mathrm{CO}$ line ratios (e.g., Casoli et al. 1992). These are almost exclusively observed in ULIRGs and were interpreted as either due to missing ${ }^{13} \mathrm{CO}$ caused by a top-heavy initial mass function in these extreme systems (Casoli et al. 1992) or due to purely optical depth effects (i.e., low ${ }^{12} \mathrm{CO}$ optical depths) caused by a warm and highly turbulent $\mathrm{H}_{2}$ medium present in these objects (Aalto et al. 1995). Then the bulk of the ${ }^{12} \mathrm{CO}$ emission arises from a diffuse enveloping phase where ${ }^{12} \mathrm{CO}$ has low to moderate optical depths $(\tau \sim 1)$, and the large ${ }^{12} \mathrm{CO} /{ }^{13} \mathrm{CO}$ ratios are due to ${ }^{13} \mathrm{CO}$-bright gas with a much a smaller filling factor than the diffuse ${ }^{12} \mathrm{CO}$-bright phase.

It is instructive to examine how far a single gas phase can go in terms of explaining the observed line ratios in Arp 220 and NGC 6240 before the fit becomes inadequate and a second gas phase must be introduced. To this end, we employ a large velocity gradient (LVG) model based on the work by Richardson (1985), which uses the observed CO line ratios to constrain the gas density $\left(n\left(\mathrm{H}_{2}\right)\right)$, kinetic temperature $\left(T_{k}\right)$, and $\Lambda_{\mathrm{CO}}=\left[\mathrm{CO} / \mathrm{H}_{2}\right](d v / d r)^{-1}$, which is the $\mathrm{CO}$ abundance relative to $\mathrm{H}_{2}$ divided by the cloud velocity gradient. The models span $n\left(\mathrm{H}_{2}\right)=10^{2}-10^{9} \mathrm{~cm}^{-3}$ (in steps of $\log n\left(\mathrm{H}_{2}\right)=0.5$ ), $T_{k}=10-100 \mathrm{~K}$ (in steps of $5 \mathrm{~K}$ ), and $\Lambda_{\mathrm{CO}}=10^{-6}$ $10^{-3}\left(\mathrm{~km} \mathrm{~s}^{-1} \mathrm{pc}^{-1}\right)^{-1}$ (in steps of $\log \Lambda_{\mathrm{CO}}=0.5$ ). The model adopts the $\mathrm{CO}-\mathrm{H}_{2}$ collisional rate constants from Flower \& Launay (1985), and included all rotational levels up to $J=11$ 
for kinetic temperatures of $10,20,40,60$, and $100 \mathrm{~K}$ were included. Collision rates for intermediate kinetic temperatures were estimated by interpolation. Finally, because the assumed ${ }^{12} \mathrm{CO} /{ }^{13} \mathrm{CO}$ abundance ratio can be important for whether a fit converges or not, we considered models with abundance ratios of 40, 60, and 100, values that span those found in the Milky Way (Langer \& Penzias 1993).

The need for a two-phase molecular ISM is perhaps most obvious in Arp 220, where no single LVG solution can account for even the ${ }^{12} \mathrm{CO} 1-0,2-1$, and $3-2$ lines. In this system, we have the interesting situation where the ratio $r_{21}={ }^{12} \mathrm{CO}$ $(2-1) /(1-0)=0.7 \pm 0.1$ points toward subthermal excitation of even ${ }^{12} \mathrm{CO} 2-1\left(n_{\text {crit }}=2.7 \times 10^{3} \mathrm{~cm}^{-3}\right)$ where typically $n\left(\mathrm{H}_{2}\right) \simeq 3 \times 10^{2} \mathrm{~cm}^{-3}$ and $T_{k} \simeq 40-60 \mathrm{~K}$ fits such a ratio well (for LTE such a low ratio implies $T_{k} \lesssim 7 \mathrm{~K}$ the minimum gas temperature permitted by cosmic-ray heating, Goldsmith \& Langer 1978). Such conditions cannot reproduce the observed $r_{32}={ }^{12} \mathrm{CO}(3-2) /(1-0)=1.0 \pm 0.1$ which indicates much more excited gas with density $\gtrsim 3 \times 10^{3} \mathrm{~cm}^{-3}$ and kinetic temperature $\gtrsim 30 \mathrm{~K}$. Going ahead to search for a LVG solution constrained by the ${ }^{12} \mathrm{CO} 1-0,2-1,3-2$ and just one ${ }^{13} \mathrm{CO}$ line strength, the $J=1-0$ lines, yields predictably a much worse solution given by $n\left(\mathrm{H}_{2}\right) \simeq 1-3 \times 10^{2} \mathrm{~cm}^{-3}$ and $T_{k} \simeq 35-45 \mathrm{~K}$. Aside from poorly reproducing the aforementioned line ratios, barely satisfying the $T_{k} \geqslant T_{\text {dust }}$ criterion for these galaxies (see 4.2), such a gas phase corresponds to brightness temperature ${ }^{13} \mathrm{CO}$ $(2-1) /(1-0),(3-2) /(1-0)$ ratios of $\mathrm{r}_{21}\left({ }^{13} \mathrm{CO}\right) \sim 1.4-1.5$ and $\mathrm{r}_{32}\left({ }^{13} \mathrm{CO}\right) \sim 0.6-0.8$, in sharp contrast to the observed ones of $\sim 1.64$ and $\sim 5$, respectively.

In the case of NGC 6240 , both $r_{21}=1.2 \pm 0.2$ and $r_{32}=1.1 \pm 0.2$ ratios indicate highly excited gas with a typical gas-phase model of $n\left(\mathrm{H}_{2}\right) \simeq 1 \times 10^{4} \mathrm{~cm}^{3}$ and $T_{k} \simeq 40-50 \mathrm{~K}$, that can also fit the observed large $J=1-0{ }^{12} \mathrm{CO} /{ }^{13} \mathrm{CO}$ ratio $\left(\mathcal{R}_{10}(\right.$ model $\left.)=37\right)$. Nevertheless this solution fails dramatically for the $J=2-1, J=3-2{ }^{12} \mathrm{CO} /{ }^{13} \mathrm{CO}$ line ratios where it yields $\mathcal{R}_{21} \sim \mathcal{R}_{32} \sim 17$, much lower than the observed values (Table 4), even for the largest plausible abundance of $\left[{ }^{12} \mathrm{CO} /{ }^{13} \mathrm{CO}\right]=100$. This could not have been found without our sensitive ${ }^{13} \mathrm{CO} 2-1$ and 3-2 measurements, which in the case of NGC 6240 , have pushed the $\mathcal{R}_{21}$ and $\mathcal{R}_{32}$ ratios to the highest such values reported in the literature.

Finally, using different $\left[{ }^{12} \mathrm{CO} /{ }^{13} \mathrm{CO}\right]$ abundance ratios namely $\left[{ }^{12} \mathrm{CO} /{ }^{13} \mathrm{CO}\right]=40,60,100$, encompassing the range deduced for the Milky Way (Langer \& Penzias 1993; Casassus et al. 2005) we still find no single set of average molecular gas physical conditions that comes even close in reproducing the ${ }^{12} \mathrm{CO}$ and the ${ }^{13} \mathrm{CO}$ line ratios in either galaxy.

\section{1. $\mathrm{C}^{18} \mathrm{O}$ Emission and the Opacity of the ${ }^{13} \mathrm{CO}$ Lines}

The emergence of ${ }^{13} \mathrm{CO}$ transitions as the first ones marking a strong deviation from any single gas phase reproducing the relative strengths of the ${ }^{12} \mathrm{CO}$ transitions is further highlighted in the case of Arp 220 by the observed strong $\mathrm{C}^{18} \mathrm{O} J=1-0$ line emission. There the $\mathrm{C}^{18} \mathrm{O} /{ }^{13} \mathrm{CO} 1-0$ line ratio of $1.0 \pm 0.3$ is the highest such ratio obtained over an entire galaxy-by comparison, Papadopoulos et al. (1996) measure such high values toward only a few of the brightest giant molecular clouds (GMCs) in NGC 1068. Assuming the low abundance of $\left[\mathrm{C}^{18} \mathrm{O} /{ }^{13} \mathrm{CO}\right]=0.15$ found for the Milky Way (Langer $\&$ Penzias 1993) such high $\mathrm{C}^{18} \mathrm{O} /{ }^{13} \mathrm{CO}$ brightness ratios argue for much of the ${ }^{13} \mathrm{CO} J=1-0$ emission emerging from a phase where $\tau_{10}\left({ }^{13} \mathrm{CO}\right)>1$, sharply deviating from the optical thin transitions deduced from the LVG of only the ${ }^{12} \mathrm{CO},{ }^{13} \mathrm{CO}$
Table 6

Line Excitation Characteristics (from Jansen 1995)

\begin{tabular}{lccc}
\hline \hline Transition & $\begin{array}{c}v_{\text {rest }} \\
(\mathrm{GHz})\end{array}$ & $\begin{array}{c}E_{u} / k_{B} \\
(\mathrm{~K})\end{array}$ & $\begin{array}{c}n_{\mathrm{crit}} \\
\left(\mathrm{cm}^{-3}\right)\end{array}$ \\
\hline${ }^{12} \mathrm{CO}(1-0)$ & 115.271 & 5.5 & $4.1 \times 10^{2}$ \\
${ }^{12} \mathrm{CO}(2-1)$ & 230.538 & 16.6 & $2.7 \times 10^{3}$ \\
${ }^{12} \mathrm{CO}(3-2)$ & 345.796 & 33.2 & $8.4 \times 10^{3}$ \\
$\mathrm{HCN}^{2}(1-0)$ & 88.632 & 4.3 & $2.3 \times 10^{5}$ \\
$\mathrm{HCN}^{2}(3-2)$ & 265.886 & 25.5 & $4.1 \times 10^{6}$ \\
$\left.\mathrm{HCN}^{2} 4-3\right)$ & 354.734 & 42.5 & $8.5 \times 10^{6}$ \\
$\mathrm{HCO}^{+}(1-0)$ & 89.189 & 4.3 & $3.4 \times 10^{4}$ \\
$\mathrm{HCO}^{+}(3-2)$ & 267.558 & 25.7 & $7.8 \times 10^{5}$ \\
$\mathrm{HCO}^{+}(4-3)$ & 356.734 & 42.8 & $1.8 \times 10^{6}$ \\
$\mathrm{CS}(2-1)$ & 97.980 & 7.1 & $8.0 \times 10^{4}$ \\
$\mathrm{CS}(3-2)$ & 146.969 & 14.1 & $2.5 \times 10^{5}$ \\
$\mathrm{CS}(5-4)$ & 244.936 & 35.3 & $1.1 \times 10^{6}$ \\
$\mathrm{CS}(7-6)$ & 342.883 & 65.8 & $2.9 \times 10^{6}$
\end{tabular}

Note. $n_{\text {crit }}=A_{u l} / \sum_{i} C_{u i}$, where $C_{u i}$ is the collisional rate "out" of the level $u$ (either up or down), calculated for $T_{k}=100 \mathrm{~K}$ in the optically thin limit.

transitions described in the previous section. A much denser gas phase can naturally yield the significantly higher optical depths needed to explain the high $\mathrm{C}^{18} \mathrm{O} /{ }^{13} \mathrm{CO} J=1-0$ ratio.

\subsection{The Properties of the Dense Molecular Gas}

In this section, we use the many transitions of $\mathrm{HCN}, \mathrm{CS}$, and $\mathrm{HCO}^{+}$in our catalog-probing a wide range of critical densities and excitation temperatures in a redundant manner (see Table 6) - to constrain the properties of the dense gas phase.

In order to model the radiative transfer of these lines, we constructed a modified version of the original LVG code. The modifications were straightforward since $\mathrm{HCN}, \mathrm{CS}$, and $\mathrm{HCO}^{+}$are simple linear molecules such as $\mathrm{CO}$. For $\mathrm{HCN}, \mathrm{CS}$, and $\mathrm{HCO}^{+}$, the collision rate constants provided by Green \& Thaddeus (1974), Turner et al. (1992), and Flower (1999) were adopted. We explored a similar density range as we did for the CO lines, but limited the temperature range to $T_{d} \leqslant T_{k} \leqslant 120 \mathrm{~K}$. The lower limit set by the dust temperature $\left(T_{d}\right)$, reflects the fact that the dust and gas are thermally decoupled, with the FUV-induced photoelectric and turbulent gas heating (and its cooling via the spectral line rather than the continuum emission) setting $T_{k} \gtrsim T_{d}$. Only in the densest, most FUVshielded and quiescent regions of the GMCs can we expect $T_{k} \simeq T_{d}$. The upper limit is the typical equilibrium temperature of purely atomic, $\mathrm{C}^{+}$-cooled $\mathrm{HI}$ in the cold neutral medium (e.g., Wolfire et al. 2003). Based on detailed fits to their FIR/ submillimeter SEDs (Dopita et al. 2005), we adopted a dust temperature of $\sim 40 \mathrm{~K}$ for both Arp 220 and NGC 6240. Since the molecules have different abundances, the range in $\Lambda$ is also different for a common velocity gradient $(d v / d r)$. In our own Galaxy, typical abundances are $\left[\mathrm{HCN} / \mathrm{H}_{2}\right] \sim$ $2 \times 10^{-8}$ (Bergin et al. 1996; Lahuis \& van Dishoeck 2000), $\left[\mathrm{CS} / \mathrm{H}_{2}\right] \sim 10^{-9}$ (Paglione et al. 1995; Shirley et al. 2003), and $\left[\mathrm{HCO}^{+} / \mathrm{H}_{2}\right]=8 \times 10^{-9}$ (Jansen 1995). In order to accommodate the substantial uncertainties associated with the abundances, the range of $\Lambda$ used was $10^{-10}-10^{-7}, 10^{-11}-10^{-8}$, and $10^{-11}$ $10^{-8}\left(\mathrm{~km} \mathrm{~s}^{-1} \mathrm{pc}^{-1}\right)^{-1}$ for $\mathrm{HCN}, \mathrm{CS}$, and $\mathrm{HCO}^{+}$, respectively. These correspond to velocity gradients in the range $\sim 0.2-$ $800 \mathrm{~km} \mathrm{~s}^{-1} \mathrm{pc}^{-1}$ for the abundances quoted above.

In the case of Arp 220, the observed HCN ratios are consistent with $\mathrm{LVG}$ solutions in the range $n\left(\mathrm{H}_{2}\right) \simeq(0.3-1) \times$ $10^{6} \mathrm{~cm}^{-3}$, and $\Lambda_{\mathrm{HCN}} \simeq(0.3-1) \times 10^{-9}\left(\mathrm{~km} \mathrm{~s}^{-1} \mathrm{pc}^{-1}\right)^{-1}$, for 
Table 7

LVG Solution Ranges, see Section 4.2 for Details

\begin{tabular}{|c|c|c|c|c|c|}
\hline Galaxy & $T_{k} /[\mathrm{K}]$ & $n\left(\mathrm{H}_{2}\right) / \mathrm{cm}^{-3}$ & $\Lambda /\left(\mathrm{km} \mathrm{s}^{-1} \mathrm{pc}^{-1}\right)^{-1}$ & $\chi_{v}^{2 \dagger}$ & $K_{\text {vir }}$ \\
\hline \multirow{2}{*}{\multicolumn{6}{|c|}{$\begin{array}{l}\text { Arp 220: } \\
\mathrm{HCO}^{+}\end{array}$}} \\
\hline & & & & & \\
\hline & $10-40$ & $(1-3) \times 10^{4}$ & $(0.3-1) \times 10^{-8}$ & $2.1-2.8$ & $0.4-0.7$ \\
\hline & $45-95$ & $1 \times 10^{4}$ & $0.3 \times 10^{-8}$ & 2.1 & 1.2 \\
\hline & $100-120$ & $0.3 \times 10^{4}$ & $1 \times 10^{-8}$ & $2.0-2.1$ & 0.7 \\
\hline \multicolumn{6}{|l|}{$\mathrm{HCN}$} \\
\hline & $10-25$ & $(0.3-1) \times 10^{6}$ & $(0.3-1) \times 10^{-7}$ & $0.5-1.3$ & $0.02-0.17$ \\
\hline & $30-40$ & $1 \times 10^{6}$ & $0.3 \times 10^{-9}$ & $0.7-0.8$ & 3.1 \\
\hline & $45-120$ & $0.3 \times 10^{6}$ & $0.1 \times 10^{-8}$ & $0.7-0.8$ & 1.7 \\
\hline \multicolumn{6}{|l|}{ CS } \\
\hline & $10-30$ & $10 \times 10^{6}$ & $(0.001-0.3) \times 10^{-8}$ & $0.2-0.5$ & $0.005-1.5$ \\
\hline & $35-40$ & $3 \times 10^{6}$ & $0.01 \times 10^{-8}$ & $0.2-0.3$ & 0.3 \\
\hline & $45-80$ & $1 \times 10^{6}$ & $0.1 \times 10^{-8}$ & $0.2-0.3$ & 0.05 \\
\hline & $85-120$ & $0.3 \times 10^{6}$ & $(0.3-1) \times 10^{-8}$ & 0.3 & $0.009-0.03$ \\
\hline \multirow{2}{*}{\multicolumn{6}{|c|}{$\begin{array}{l}\text { NGC 6240: } \\
\mathrm{HCO}^{+}\end{array}$}} \\
\hline & & & & & \\
\hline & $10-25$ & $(1-3) \times 10^{4}$ & $(0.3-1) \times 10^{-8}$ & $1.9-2.7$ & $0.4-0.7$ \\
\hline & $30-55$ & $1 \times 10^{4}$ & $0.3 \times 10^{-8}$ & $1.8-1.9$ & 1.2 \\
\hline & $60-120$ & $0.3 \times 10^{4}$ & $1 \times 10^{-8}$ & $1.6-1.7$ & 0.7 \\
\hline \multicolumn{6}{|l|}{$\mathrm{HCN}$} \\
\hline & $10-20$ & $(0.1-1) \times 10^{6}$ & $(0.3-1) \times 10^{-7}$ & $1.3-1.7$ & $0.03-0.1$ \\
\hline & $25-30$ & $0.3 \times 10^{6}$ & $0.1 \times 10^{-8}$ & $1.3-1.4$ & 1.7 \\
\hline & $35-55$ & $0.1 \times 10^{6}$ & $1 \times 10^{-8}$ & $1.3-1.5$ & 0.3 \\
\hline & $60-120$ & $0.3 \times 10^{6}$ & $0.03 \times 10^{-8}$ & $1.4-1.5$ & 5.5 \\
\hline
\end{tabular}

Notes. The most likely solutions have been highlighted in bold.

$\dagger$ the chi-squared fit was calculated as $\chi_{v}^{2}=\Sigma_{i}{\frac{1}{\sigma_{i}}}^{2}\left(R_{\mathrm{obs}}-R_{\text {model }}\right)^{2}$, where $R_{\mathrm{obs}}$ and $R_{\text {model }}$ are the observed and modeled line ratios, respectively, and $\sigma_{i}$ is the associated error.

which $\tau_{\mathrm{HCN}(1-0)} \simeq 3-7$. From the fit of the CS lines we find $n\left(\mathrm{H}_{2}\right) \simeq(0.3-3) \times 10^{6} \mathrm{~cm}^{-3}$, and $\Lambda_{\mathrm{CS}} \simeq(0.01-1) \times$ $10^{-8}\left(\mathrm{~km} \mathrm{~s}^{-1} \mathrm{pc}^{-1}\right)^{-1}$. Finally, the subthermal $\mathrm{HCO}^{+}$ratios allow for solutions in the range $n\left(\mathrm{H}_{2}\right) \simeq(0.3-1) \times 10^{4} \mathrm{~cm}^{-3}$, and $\Lambda_{\mathrm{HCO}^{+}} \simeq(0.3-1) \times 10^{-8}\left(\mathrm{~km} \mathrm{~s}^{-1} \mathrm{pc}^{-1}\right)^{-1}$. All the aforementioned solutions are for $T_{k} \geqslant 40 \mathrm{~K}$ and their details are shown in Table 7. There it can be seen that while $\mathrm{HCN}, \mathrm{CS}$, and $\mathrm{HCO}^{+}$ all trace what in broad terms can be characterized as dense gas (i.e., $n\left(\mathrm{H}_{2}\right) \gtrsim 10^{4} \mathrm{~cm}^{-3}$ ), with the density range of the $\mathrm{CS}$ and HCN solutions being very similar. Moreover we find the CS and HCN emission tracing gas with densities $\sim 100$ times higher than that traced by the $\mathrm{HCO}^{+}$lines. This simply reflects the high $\mathrm{HCN}, \mathrm{CS}$ and the low $\mathrm{HCO}^{+}$line ratios measured in Arp 220, further reinforced by the $\sim$ five times lower critical densities of the $\mathrm{HCO}^{+}$transitions than, e.g., those of $\mathrm{HCN}$ at the same rotational level (see Table 6). These results are certainly consistent with Galactic surveys of CS lines, which have shown that they are unmistakable markers of very dense, high-mass star-forming cores (Plume et al. 1997; Shirley et al. 2003).

For NGC 6240, the HCN LVG fits converge mostly over $n\left(\mathrm{H}_{2}\right) \simeq(1-3) \times 10^{5} \mathrm{~cm}^{-3}, T_{k} \geqslant 40 \mathrm{~K}$, and $\Lambda_{\mathrm{HCN}} \simeq 3 \times$ $10^{-10}-1 \times 10^{-8}\left(\mathrm{~km} \mathrm{~s}^{-1} \mathrm{pc}^{-1}\right)^{-1}$, with an optically thick HCN $J=1-0$ line $\left(\tau_{\mathrm{HCN}(1-0)} \simeq 3-29\right)$. From the $\mathrm{HCO}^{+}$lines we find solutions in the range $n\left(\mathrm{H}_{2}\right) \simeq(0.3-1) \times 10^{4} \mathrm{~cm}^{-3}$, $T_{k} \geqslant 40 \mathrm{~K}$, and $\Lambda_{\mathrm{HCO}^{+}} \simeq(0.3-1) \times 10^{-8}\left(\mathrm{~km} \mathrm{~s}^{-1} \mathrm{pc}^{-1}\right)^{-1}$. Thus in NGC 6240, as in Arp 220, HCN is tracing gas which is significantly denser than that traced by $\mathrm{HCO}^{+}$, over a similar range of temperatures.

In an attempt to "break" the significant degeneracy that still exists in the LVG solutions for the dense gas we make use of the fact that at least in the Galaxy, most of the dense star-forming gas is found in self-gravitating cores (Shirley et al. 2003). In the cases of Arp 220 and NGC 6240, we use this to try and limit the allowed $\left(n\left(\mathrm{H}_{2}\right), T_{k}, \Lambda_{\mathrm{X}}\right)$-parameter space by examining the ratio of the velocity gradient inferred from the $\mathrm{LVG}$ fits to that expected for virialized cores, namely

$$
K_{\mathrm{vir}}=\frac{(d v / d r)_{\mathrm{LVG}}}{(d v / d r)_{\mathrm{vir}}} \sim 1.54 \frac{\left[\mathrm{X} / \mathrm{H}_{2}\right]}{\sqrt{\alpha} \Lambda_{X}}\left(\frac{n\left(\mathrm{H}_{2}\right)}{10^{3} \mathrm{~cm}^{-3}}\right)^{-1 / 2},
$$

where $\left[\mathrm{X} / \mathrm{H}_{2}\right]$ is the abundance ratio of the given molecule $\mathrm{X}$ (see Papadopoulos \& Seaquist 1999, or Goldsmith 2001 for a derivation), and $\alpha \sim 1-2.5$ is a constant whose value depends on the assumed density profile of a typical cloud (Bryant \& Scoville 1996).

For virialized gas we expect $K_{\mathrm{vir}} \sim 1$ to within a factor of a few (due to uncertainties in cloud geometry, density profile, and abundance assumed to deduce the observed $d v / d r$ from $\Lambda_{X}$ ). LVG solutions that correspond to $K_{\text {vir }}>>1$ indicate nonvirial, unbound motions and are ruled out for dense star-forming gas along with those that have $K_{\text {vir }} \ll 1$ (gas motions cannot be slower than those dictated by its self-gravity). For Arp 220 and Table 7 we see that for both $\mathrm{HCO}^{+}$and $\mathrm{HCN}, \mathrm{LVG}$ solutions with $T_{k} \geqslant 40 \mathrm{~K}$ have $K_{\text {vir }} \simeq 1$, and thus these conditions do not single out any particular range of solutions. For CS however, we find $K_{\mathrm{vir}} \ll 1$ for most solutions except for $T_{k}=35-40 \mathrm{~K}$, $\mathrm{n}\left(\mathrm{H}_{2}\right)=3 \times 10^{6} \mathrm{~cm}^{-3}$ and $\Lambda_{\mathrm{CS}}=10^{-10}\left(\mathrm{~km} \mathrm{~s}^{-1} \mathrm{pc}^{-1}\right)^{-1}$. This suggests that the true CS abundance may be significantly higher than assumed (by at least an order of magnitude). It should be noted, however, that other studies have found evidence for CS abundances close to $10^{-8}$ in Galactic star-forming cores (Hatchell \& van der Tak 2003). Adopting this value would result in $K_{\text {vir }}$ values closer to unity. Finally for NGC 6240 all solutions that fit the $\mathrm{HCO}^{+}$ratios are consistent with $K_{\text {vir }} \sim 1$, while for $\mathrm{HCN}$ this is the case for $T_{k}=40-55 \mathrm{~K}$ and $n\left(\mathrm{H}_{2}\right)=10^{5} \mathrm{~cm}^{-3}$ and $\Lambda_{\mathrm{HCN}}=10^{-8}\left(\mathrm{~km} \mathrm{~s}^{-1} \mathrm{pc}^{-1}\right)^{-1}$ (see Table 7).

\subsubsection{HNC versus $H C N$}

Observations of HCN and HNC in Galactic GMCs (Goldsmith et al. 1981), as well as steady-state chemical models (Schilke et al. 1992), predict an increase in the $\mathrm{HCN} / \mathrm{HNC}$ abundance ratio with increasing temperature and density, and attribute this to the destruction of $\mathrm{HNC}$ (but not $\mathrm{HCN}$ ) via neutralneutral reactions with atomic oxygen or hydrogen. Then, at densities $\sim 10^{4-5} \mathrm{~cm}^{-1}$, temperature-independent ion-neutral reactions, which form $\mathrm{HCN}$ and $\mathrm{HNC}$ in equal amounts, may become dominant (Schilke et al. 1992; Aalto et al. 2002), and as a result the $\mathrm{HNC}$ abundance can be substantial. However, given the high optical depths implied for the HCN $J=1-0$ transition from our excitation analysis of the $\mathrm{HCN}$ line ratios, no useful limits can be placed on the $[\mathrm{HNC} / \mathrm{HCN}]$ abundance ratio from the observed brightness temperature ratio of $1.2 \pm 0.2$ measured for their $J=1-0$ transition in Arp 220 (consistent with both lines being optically thick). For a comparative analysis of $\mathrm{HCN}$ versus $\mathrm{HNC}$ excitation using several rotational transitions, and the diagnostic nature of the $[\mathrm{HNC} / \mathrm{HCN}]$ abundance ratio for the excitation environment of ULIRGs the interested reader is referred to recent work by Aalto et al. (2007).

\subsection{Subthermal $\mathrm{HCO}^{+}$Line Excitation: $\mathrm{HCO}^{+}$as Dense Gas Mass Tracer}

In both galaxies the low $\mathrm{HCO}^{+}$line ratios result in $\mathrm{LVG}$ solutions with densities mostly $\sim 10-100$ times lower than those 
Table 8

Estimates of the Total Dense Gas Mass in Arp 220 and NGC 6240

\begin{tabular}{lcc}
\hline \hline Method & \multicolumn{2}{c}{$M_{\text {dense }}\left[\times 10^{10} M_{\odot}\right]$} \\
\hline Method 1 & Arp 220 & NGC 6240 \\
$\mathrm{HCO}^{+}(1-0)$ & & \\
$\mathrm{HCN}(1-0)$ & $0.5-1.6$ & $1.3-2.2$ \\
$\mathrm{CS}(2-1)$ & $1.8-4.2$ & $1.0-2.8$ \\
Method 2 & $0.3-1.5$ & $\ldots$ \\
HCN & & \\
Best Estimate & $1.4-7.0$ & $1.7-5.7$ \\
\hline
\end{tabular}

Note. See Section 1.4 for details.

deduced from the analysis of the HCN ratios (Table 7). Thus if only $\mathrm{HCO}^{+}$line observations were used, they would imply a bulk dense gas phase dominated by less extreme densities than is actually the case, especially in Arp 220. The several $\mathrm{HCO}^{+}$and $\mathrm{HCN}$ transitions observed, and the independent analysis afforded by the CS transitions (for Arp 220), make it unlikely that the aforementioned conclusions can be attributed to enhanced HCN abundances (and thus thermalization of its transitions at lower densities because of radiative trapping) caused by XDRs, or simply to IR-pumping of $\mathrm{HCN} J=1-0$. Thus recent claims that $\mathrm{HCO}^{+}$is a better tracer of the dense gas in (U)LIRGs than HCN (Graciá-Carpio et al. 2006), do not seem to hold in the cases of Arp 220 and NGC 6240, and a more comprehensive approach is needed to decide the case (Papadopoulos 2007).

\subsection{The Mass of the Dense Gas Phase in Arp 220 and NGC 6240}

In this section, we make use of the constraints on the dense gas in Arp 220 and NGC 6240 obtained in Section 4.2 to derive the total mass of the dense gas in these two systems. We do this using two different methods, the results of which are summarized in Table 8 . The first one assumes the dense gas reservoir to be fully reducible to an ensemble of self-gravitating units whose molecular line emission does not suffer any serious cloud-cloud shielding. Then it can be shown (e.g., Dickman et al. 1986) that the total mass of, e.g., an HCN-luminous dense gas phase can be found from

$$
M_{\text {dense }}\left(\mathrm{H}_{2}\right)=\alpha_{\mathrm{HCN}} \mathrm{L}_{\mathrm{HCN}}^{\prime},
$$

where $\alpha_{\mathrm{HCN}} \simeq 2.1 \sqrt{n\left(\mathrm{H}_{2}\right)} / T_{b, \mathrm{HCN}(1-0)}$ is a conversion factor (e.g., Radford et al. 1991a). We insert the range of $n\left(\mathrm{H}_{2}\right)$ and $T_{b, \mathrm{HCN}(1-0)}$ values inferred from the LVG solutions to the $\mathrm{HCN}$ line ratios, which for Arp 220 are $n\left(\mathrm{H}_{2}\right)=0.3 \times 10^{6} \mathrm{~cm}^{-3}$ and $T_{b, \mathrm{HCN}(1-0)}=34-62 \mathrm{~K}$, and for NGC $6240 n\left(\mathrm{H}_{2}\right)=$ $(0.1-0.3) \times 10^{6} \mathrm{~cm}^{-3}$ and $T_{b, \mathrm{HCN}(1-0)}=31-40 \mathrm{~K}$. From these values we find $\alpha_{\mathrm{HCN}} \simeq(19-34) M_{\odot}\left(\mathrm{K} \mathrm{km} \mathrm{s}^{-1} \mathrm{pc}^{2}\right)^{-1}$ and $\alpha_{\mathrm{HCN}} \simeq(17-37) M_{\odot}\left(\mathrm{K} \mathrm{km} \mathrm{s}^{-1} \mathrm{pc}^{2}\right)^{-1}$ for Arp 220 and NGC 6240, which in turn yields dense gas mass traced by $\mathrm{HCN}$ of $M_{\text {dense }}\left(\mathrm{H}_{2}\right) \simeq(1.8-4.2) \times 10^{10} M_{\odot}$ and $\simeq(1.0-2.8) \times$ $10^{10} M_{\odot}$ for these two systems respectively.

In a similar fashion, the analysis of $\mathrm{HCO}^{+}$and $\mathrm{CS}$ line ratios with their corresponding $T_{b}$ and $n\left(\mathrm{H}_{2}\right)$ values yields corresponding conversion factors which, along with the luminosities of the lowest $\mathrm{HCO}^{+}$and $\mathrm{CS}$ transitions available, yield the dense gas mass traced by each species (Table 8). The constraints of an underlying density-size cloud hierarchy (see Section 4.5) demand $M\left(\mathrm{H}_{2}\right)_{\mathrm{HCN} / \mathrm{CS}} \leqslant M_{\mathrm{HCO}^{+}}$(since the $\mathrm{HCN}, \mathrm{CS}$ lines are tracing a denser gas than those of $\left.\mathrm{HCO}^{+}\right)$. Thus from
Table 8 we obtain $M_{\text {dense }}\left(\mathrm{H}_{2}\right) \sim 1.5 \times 10^{10} \mathrm{M}_{\odot}($ Arp 220$)$ and $M_{\text {dense }}\left(\mathrm{H}_{2}\right) \sim(1-2) \times 10^{10} \mathrm{M}_{\odot}(\mathrm{NGC} 6240)$ as the best dense gas-mass estimates conforming to the aforementioned inequality. Systematic biases can be introduced by (1) the lack of a pressure-term in Equation (6) correcting for molecular gas overlying the dense gas regions, (2) by the existence of substantial stellar mass even within the dense gas phase. In both of these cases the true conversion factor and the deduced gas masses will be lower (see Bryant \& Scoville 1996; Downes \& Solomon 1998 for the appropriate formalism).

Finally, given the significant number of $\mathrm{HCN}$ transition available for both systems, we can estimate the dense gas mass by assuming the bulk of the HCN molecules are in rotational states $J \leqslant 6$. Then

$$
\begin{aligned}
M_{\text {dense }}\left(\mathrm{H}_{2}\right)= & {\left[\mathrm{H}_{2} / \mathrm{HCN}\right]\left[\mathrm{N}_{1}+\mathrm{N}_{2}+\mathrm{N}_{3}+\mathrm{N}_{4}+\mathrm{N}_{5}\right.} \\
& \left.+N_{6}\right] \mu m_{\mathrm{H}_{2}},
\end{aligned}
$$

where $N_{J}$ is the total number of HCN molecules in state $J$ and $\mu=1.36$ accounts for He. The $\mathrm{HCN}$ population numbers are calculated from

$$
N_{J}=\frac{8 \pi k v_{J, J-1}^{2}}{h c^{3} A_{J, J-1}} \beta_{J, J-1}^{-1} L_{\mathrm{HCN}(J, J-1)}^{\prime},
$$

where $A_{J, J-1}$ and $\beta_{J, J-1}=\left(1-e^{-\tau_{J, J-1}}\right) / \tau_{J, J-1}$ are the Einstein coefficient and the escape probability for the $J \rightarrow J-1$ transition, respectively. Unlike the first method this approach depends directly on the assumed $\left[\mathrm{HCN} / \mathrm{H}_{2}\right]$ abundance ratio while it is in principle independent of any assumptions regarding the velocity field of the dense gas phase (i.e., it remains valid for a nonvirialized, unbound gas phase), as long as the $\mathrm{HCN}$ line emission remains effectively optically thin (i.e., any large $\tau$ 's emerge within small gas "cells"). The dense gas masses estimated with the second method (see Table 8) bracket the best estimates yielded by the first one (constrained by the densitymass hierarchy assumption), though more tightly for Arp 220 than NGC 6240.

The two methods overlap over the same range of $M\left(\mathrm{H}_{2}\right)_{\text {dense }} \sim(1-2) \times 10^{10} \mathrm{M}_{\odot}$ for both galaxies, which is comparable to the total molecular gas mass estimated independently from models of their interferometrically imaged $\mathrm{CO}$ emission (Downes \& Solomon 1998), or their CI $J=1-0$ fluxes (Papadopoulos \& Greve 2004). Thus the bulk of the molecular ISM in these two extreme starbursts is in a dense state with $n\left(H_{2}\right) \sim\left(10^{5}-10^{6}\right) \mathrm{cm}^{-3}$.

It is worth comparing this mass to the fundamental upper limit set by the dynamical mass in these objects. For Arp 220, the latter was estimated to be $M_{\text {dyn }} \simeq 4 \times 10^{10} M_{\odot}$ (adopted to the cosmology used here) within a radius of $1.4 \mathrm{kpc}$, based on resolved observations of CO (Downes \& Solomon 1998). For NGC $6240 M_{\text {dyn }} \simeq(0.7-1.6) \times 10^{10} M_{\odot}$ within a radius of $\sim$ 500 pc (Tacconi et al. 1999; Bryant \& Scoville 1999). The COemitting region in NGC 6240 extends significantly beyond that (Tacconi et al. 1999) and thus a significant amount of molecular gas may reside beyond the inner 500 pc. Assuming a constant rotation curve, we obtain an upper limit of $M_{\text {dyn }}=6 \times 10^{10} M_{\odot}$ within $R=1.4 \mathrm{kpc}$ for NGC 6240 . Since any $\mathrm{HCN} / \mathrm{CS} / \mathrm{HCO}^{+}$ bright region will be contained within the $\mathrm{CO}$-bright regions we expect $M_{\text {dyn }} \geqslant M_{\mathrm{CO}} \geqslant M_{\text {dense }}$ in both systems. Thus the range of $M\left(\mathrm{H}_{2}\right)_{\text {dense }} \sim(1-2) \times 10^{10} \mathrm{M}_{\odot}$ corresponds to $\sim 20 \%-50 \%$ of the dynamical mass in Arp 220, and $\gtrsim 15 \%$ up to $100 \%$ for NGC 6240. 


\subsection{The Density-Size Relation for the Dense Gas: Steeper than Larson's Law?}

In this section, we attempt to go a step beyond using $\mathrm{HCO}^{+}$, $\mathrm{HCN}$, and CS lines as isolated estimators of the dense gas mass by assuming their emission emerging from an underlying density-size hierarchy, found for Galactic molecular clouds by Larson (1981), and verified by subsequent high-resolution multitransition studies. The origin of the density-size and linewidth-size power laws revealed for GMCs in the Galaxy may lie in the characteristics of supersonic turbulence and its global driving mechanisms (Heyer \& Brunt 2004) when present on self-gravitating structures. As such they may remain similar even in the extreme ISM conditions present in ULIRGs.

A rigorous approach to test this in extragalactic environments (where molecular clouds cannot be viewed with the same level of detail) using the global molecular line emission of various species would require incorporating such power laws in the radiative transfer model used to interpret the emergent molecular line emission. In our case, we opt for a simpler approach in which the denser HCN-bright gas is simply assumed to be "nested" within the less-dense regions traced by the $\mathrm{HCO}^{+}$transitions.

We can rudimentary test for this by deriving the velocityaveraged area-filling factor of the $\mathrm{HCN}$-bright relative to the $\mathrm{HCO}^{+}$-bright gas phase: $f_{\mathrm{HCN}, \mathrm{HCO}^{+}}$, which ought to be $<1$. This can be found from

$$
\frac{L_{\mathrm{HCN}}^{\prime}}{L_{\mathrm{HCO}^{+}}^{\prime}}=f_{\mathrm{HCN}, \mathrm{HCO}^{+}} \frac{T_{b, \mathrm{HCN}}}{T_{b, \mathrm{HCO}^{+}}} .
$$

For the LVG solution ranges above $\mathrm{T}_{\mathrm{k}}=40 \mathrm{~K}$ (Table 7) and the $J=1-0$ transition of both species, the observed line luminosities and LVG-deduced intrinsic brightness temperatures yield: $0.2 \lesssim f_{\mathrm{HCN}, \mathrm{HCO}^{+}} \lesssim 1.0($ Arp 220$)$ and $0.1 \lesssim f_{\mathrm{HCN}, \mathrm{HCO}^{+}} \lesssim$ 0.3 (NGC 6240). For an underlying density-size power law $\left\langle\mathrm{n}\left(\mathrm{H}_{2}\right)\right\rangle \propto \mathrm{R}^{-\alpha}$, we set $\alpha=2 \log \left(n_{\mathrm{HCO}^{+}} / n_{\mathrm{HCN}}\right) / \log f_{\mathrm{HCN}, \mathrm{HCO}^{+}}$ as an approximation $\left(n_{\mathrm{HCN}}, n_{\mathrm{HCO}^{+}}\right.$are the mean volume densities deduced from the $\mathrm{LVG}$ fits of the $\mathrm{HCN}$ and $\mathrm{HCO}^{+}$ line ratios). For the range of densities corresponding to LVG fits of the $\mathrm{HCN}$ and $\mathrm{HCO}^{+}$ratios (for $T_{k} \geqslant 40 \mathrm{~K}$ ), we find $\alpha \gtrsim 2$, steeper than the value of $\alpha \sim 1$ typical for Galactic molecular clouds. Using the $\mathrm{HCO}^{+}$and $\mathrm{HCN} J=4-3$ transitions instead yields $0.09 \lesssim f_{\mathrm{HCN} \mathrm{HCO}^{+}} \lesssim 0.6$ (Arp 220), and $0.03 \lesssim f_{\mathrm{HCN}, \mathrm{HCO}^{+}} \lesssim 0.2$ (NGC6240). These smaller values are expected for emission from the two lines bracketing the entire range critical densities in our line inventory (Table 6), and an underlying density-size ISM hierarchical structure. However, even for these values of $f_{\mathrm{HCN}, \mathrm{HCO}^{+}}$the corresponding density ranges yield $\alpha \gtrsim 1.3$, still steeper than a Larson-type power law. Such a steepening could in principle be the result of extreme tidal stripping of GMCs and then merging of the surviving densest clumps to superdense structures, yet another signature of vastly different average molecular gas properties in extreme starbursts than those in more quiescent environments.

\subsection{The Diffuse Gas Phase}

The observed ${ }^{12} \mathrm{CO}$ line emission will have contributions from both the dense and diffuse gas and the observed global line ratio of any given $J+1 \longrightarrow J$ line with respect to ${ }^{12} \mathrm{CO} 1-0$ can therefore be written as

$$
r_{J+1, J}=\frac{r_{J+1, J}^{(A)}+C_{10}^{(12)} r_{J+1, J}^{(B)}}{1+C_{10}^{(12)}},
$$

where $r_{J+1, J}^{(A)}$ is the line ratio for the diffuse phase (denoted by A), $r_{J+1, J}^{(B)}$ the one for the dense phase (denoted by B), and the contrast factor, $C_{10}^{(12)}$ is discussed below. It is seen from Equation (10) that in order to constrain the properties of the diffuse gas, one has to determine and subtract the contribution from the dense gas phase. Fortunately, this can now be done with the constraints put on the dense phase properties in Section 4.2. The contrast factor, which determines the relative emission contribution of the two gas phases, is given by $C_{10}^{(12)}=f_{B A}\left(T_{b, 10}^{(B)} / T_{b, 10}^{(A)}\right)$, where $f_{B A}$ is the velocity-integrated relative filling factor between the two phases and $T_{b, 10}^{A, B}$ are the velocity- and area-integrated line brightness temperatures of ${ }^{12} \mathrm{CO} 1-0$. In order to determine this quantity, we make the reasonable assumption that the $\mathrm{HCN}$ emission is dominated by the dense gas phase and, as a result, we can write the global (observed) $\mathrm{HCN}(1-0) /{ }^{12} \mathrm{CO}(1-0)$ line ratio as

$$
\frac{T_{b, \mathrm{HCN}}}{T_{b, \mathrm{CO}}}=\frac{C_{10}^{(12)}}{1+C_{10}^{(12)}} \frac{T_{b, \mathrm{HCN}}^{(B)}}{T_{b, \mathrm{CO}}^{(B)}} .
$$

We then estimate $C_{10}^{(12)}$ using the observed value of $T_{b, \mathrm{HCN}} / T_{b, \mathrm{CO}}$ and the $T_{b, \mathrm{HCN}}^{(B)} / T_{b, \mathrm{CO}}^{(B)}$ values obtained from the LVG fits of the dense gas phase. The observed global line ratio for Arp 220 is $T_{b, \mathrm{HCN}} / T_{b, \mathrm{CO}}=0.18 \pm 0.03$, and $T_{b, \mathrm{HCN}}^{(B)} / T_{b, \mathrm{CO}}^{(B)}=0.5-0.8$ as estimated from the dense gas solution range. In the case of NGC 6240, the same two line ratios are $0.08 \pm 0.01$ and $0.4-0.8$. Folding in the uncertainties on the observed global ratios and allowing for the range in the ratio predicted for the dense gas, we find $C_{10}^{(12)} \sim 1.5-3.0\left(\right.$ Arp 220) and $C_{10}^{(12)} \sim 1.3-2.8$ (NGC 6240).

With $C_{10}^{(12)}$ determined, we can then disentangle the contributions to the ${ }^{12} \mathrm{CO}$ line ratios from the diffuse and dense phases using Equation (10). If we assume the $\mathcal{R}$ ratios dominated by the diffuse phase, the resulting ${ }^{12} \mathrm{CO}$ and ${ }^{13} \mathrm{CO}$ emission-line ratios for Arp 220 are consistent with $n\left(\mathrm{H}_{2}\right) \simeq$ $(1-3) \times 10^{2} \mathrm{~cm}^{-3}, T_{k} \geqslant 40 \mathrm{~K}$, and $\Lambda_{\mathrm{CO}} \simeq(3-10) \times$ $10^{-5}\left(\mathrm{~km} \mathrm{~s}^{-1} \mathrm{pc}^{-1}\right)^{-1}$. For NGC 6240, the LVG model converges on $n\left(\mathrm{H}_{2}\right) \simeq(1-3) \times 10^{3} \mathrm{~cm}^{-3} T_{k} \geqslant 40 \mathrm{~K}$, and $\Lambda_{\mathrm{CO}} \simeq(1-3) \times 10^{-6}\left(\mathrm{~km} \mathrm{~s}^{-1} \mathrm{pc}^{-1}\right)^{-1}$. All solutions correspond to nonvirialized $K_{\text {vir }} \gtrsim 5$ values (assuming a Galactic abundance $\left[\mathrm{CO} / \mathrm{H}_{2}\right]=10^{-4}$ ) and have moderate ${ }^{12} \mathrm{CO} J=1-0$ optical depths $\left(\tau_{10} \sim 1\right)$. This seems to be the typical diffuse gas phase found in such systems (e.g., Aalto et al. 1995; Downes \& Solomon 1998) which, while dominating their low-J CO line emission, it does not contain much of their total molecular gas mass.

\section{DENSE GAS MASS, STAR FORMATION, AND ITS EFFICIENCY IN Arp 220 AND NGC 6240}

The dense gas masses derived for Arp 220 and NGC 6240 in Section 4.4, allow us to derive accurate SFEs in these two galaxies. Although the SFE was originally defined as $\mathrm{SFE}=\mathrm{SFR} / M\left(\mathrm{H}_{2}\right)$, where SFR is the SFR and $M\left(\mathrm{H}_{2}\right)$ is the total molecular gas mass, we shall here focus on the SFE per dense gas mass, with the SFR parameterized by the IR luminosity (i.e., $\mathrm{SFE} \propto L_{\mathrm{IR}} / M_{\text {dense }}$ ).

Using the definition of IR luminosity as the SED integrated from $40 \mu \mathrm{m}$ to $120 \mu \mathrm{m}$, we derive $L_{\mathrm{IR}}=1.6 \times 10^{12} L_{\odot}$ (Arp 220) and $L_{\mathrm{IR}}=7.0 \times 10^{11} L_{\odot}(\mathrm{NGC} \mathrm{6240)}$. For the deduced dense gas-mass range of $M_{\text {dense }}\left(\mathrm{H}_{2}\right) \sim(1-2) \times 10^{10} \mathrm{M}_{\odot}$ 
we obtain $L_{\mathrm{IR}} / M_{\text {dense }} \sim(80-160) L_{\odot} M_{\odot}{ }^{-1}$ (Arp 220) and $L_{\mathrm{IR}} / M_{\text {dense }}=(35-70) L_{\odot} M_{\odot}{ }^{-1}$ (NGC 6240), suggesting a somewhat higher SFE in Arp 220. Finally these values are within the range of those found for a large sample of IR galaxies, consisting of large spiral galaxies, LIRGs, and a few ULIRG by Gao \& Solomon (2004a, 2004b) using the HCN 1-0 line as a linear tracer of dense gas.

A density-size hierarchy found for Galactic GMCs remaining valid in other galaxies would suggest that $\mathrm{HCO}^{+}, \mathrm{HCN}$, and CS lines, given their increasing excitation requirements, would probe progressively deeper into the warm and dense gas phase fuelling star formation. We briefly explore this scenario by calculating three separate star formation efficiencies, $\mathrm{SFE}_{\mathrm{HCO}^{+}}$, $\mathrm{SFE}_{\mathrm{HCN}}$, and $\mathrm{SFE}_{\mathrm{CS}}$, using the separate dense gas-mass estimates based on $\mathrm{HCO}^{+}, \mathrm{HCN}$, and CS available for Arp 220 only. For this galaxy we find $\operatorname{SFE}_{\mathrm{CS}}\left(\simeq 107-533 L_{\odot} M_{\odot}{ }^{-1}\right)$ is larger than $\mathrm{SFE}_{\mathrm{HCN}}\left(\simeq 40-94 L_{\odot} M_{\odot}{ }^{-1}\right)$, which may suggest that the star formation increases as we probe higher densities (Shirley et al. 2003). On the other hand, this trend is not confirmed by $\mathrm{HCO}^{+}$, which yields $\mathrm{SFE}_{\mathrm{HCO}^{+}} \simeq 100$ $320 L_{\odot} M_{\odot}^{-1}$, just as we do not see a significantly narrower range in SFE values at higher densities as one might otherwise expect (Shirley et al. 2003). In NGC 6240 the SFE derived from $\mathrm{HCO}^{+}\left(\mathrm{SFE}_{\mathrm{HCO}^{+}} \simeq 32-54 L_{\odot} M_{\odot}{ }^{-1}\right)$ is smaller than the efficiency obtained from $\mathrm{HCN}\left(\mathrm{SFE}_{\mathrm{HCN}} \simeq 41-70 L_{\odot} M_{\odot}{ }^{-1}\right)$. We conclude that given the uncertainties in the mass estimates we cannot robustly claim a significant increase in the SFEs derived using $\mathrm{HCO}^{+}, \mathrm{HCN}$, and $\mathrm{CS}$.

Theory currently provides some hints that support a small range of SFEs per dense gas mass. Simulations of star formation in turbulent media where dense $\left(n\left(\mathrm{H}_{2}\right)>10^{4} \mathrm{~cm}^{-3}\right)$ gas clumps decouple from a supersonic, turbulent cascade to form stars at a constant efficiency seem to reproduce well some of the observed characteristics of SFR versus gas in galaxies (Krumholz \& McKee 2005). Furthermore, Scoville (2004) showed that the negative feedback effect of high-mass star formation naturally leads to an upper bound on the SFE for star-forming regions of $500 L_{\odot} M_{\odot}{ }^{-1}$. Although the exact value of this upper limit may change in the future (since it depends on the poorly constrained effective radiative opacity of the dust), the underlying physics is expected to apply to local star-forming regions as well as those in circumnuclear starbursts. We note that our derived SFEs are consistent with the current upper limit.

\section{IMPLICATIONS FOR DENSE STAR FORMING GAS AT HIGH REDSHIFTS}

Evidence from Galactic observations and recent results from the ULIRG/QSO Mrk 231 (Papadopoulos et al. 2007b) point to the same gas phase responsible for both $\mathrm{HCN}$ and high- $J \mathrm{CO}$ line emission. This is expected, as such a phase is intimately linked with ongoing star formation and thus its warm and dense conditions will excite the high- $J \mathrm{CO}$ transition and those of $\mathrm{HCN}, \mathrm{CS}$ and $\mathrm{HCO}^{+}$alike. Thus the weaker lines of heavy rotor molecules with frequencies within the numerous transparent atmospheric windows at $\lesssim 350 \mathrm{GHz}$, can be used to probe the gas emitting also the luminous CO $J+1 \rightarrow J, J+1 \geqslant 4$ lines at frequencies of $v \gtrsim 460 \mathrm{GHz}$ beyond which the atmospheric transparency window rapidly closes, even in sites where prime submillimeter telescopes are located.

In Figures 3(a) and (c), we have plotted the expected line fluxes of the ${ }^{12} \mathrm{CO}$ and ${ }^{13} \mathrm{CO}$ line templates-based on Arp 220 and NGC 6240, respectively-for redshifts $z=0.05,0.1,0.5$, and 1.0. The ${ }^{12} \mathrm{CO}$ and ${ }^{13} \mathrm{CO}$ line fluxes were extracted from the LVG model with input conditions set by the HCN solution ranges for the dense gas in these two systems (Table 7). Similarly, Figures 3(b) and (d) show the $\mathrm{HCN} / \mathrm{CS} / \mathrm{HCO}^{+}$line templates for the solution ranges given in Table 7 . For comparison we also show the latest sensitivity estimates for the HI-FI instrument (de Graauw et al. 1998, 2005) — a series of highly advanced heterodyne receivers due to fly onboard Herschel, an ESA cornerstone mission expected to launch in 2008. HI-FI will observe in six bands covering the frequency range $480-1910 \mathrm{GHz}$, enabling it to target the highest $J$-transitions in low- $z$ sources. Figure 3 shows that if Arp 220 and NGC 6240 are not significantly different from the bulk population of (U)LIRGs in the redshift range $0 \leqslant z \leqslant 1$, HI-FI/Herschel will be able to detect the $\mathrm{CO} J \rightarrow J-1, J \geqslant 5$ lines in such objects out to $z \sim 0.1$ in less than 1 h. At $z \sim 0.5$ the $\mathrm{CO} J \rightarrow J-1, J \geqslant 7$ lines are still within the HI-FI frequency range, and are easily detectable. We see the $\mathrm{HCN}$ and $\mathrm{HCO}^{+} J \rightarrow J-1, J \geqslant 6$ lines are detectable in systems akin to Arp 220 and NGC 6240 out to $z \sim 0.1$. At $z \sim 0.5$, however, $\mathrm{HCN}$ lines are still detectable only in Arp 220-like systems. The predicted $\mathrm{HCO}^{+}$lines fluxes for both types of systems at $z \sim 0.5$ fall below the $1 \mathrm{~h} 5 \sigma$ sensitivity limits of $\mathrm{HI}-\mathrm{FI}$.

Thus for (U)LIRGs in the redshift range $0 \leqslant z \lesssim 0.5$, HI-FI will complement ground-based (sub)millimeter facilities where only the low- $J$ lines are accessible and thereby densely sample the rotational ladder for a number of important molecules in such systems. This will allow a full inventory of the molecular ISM in luminous starbursts and (U)LIRGs and provide a valuable set of low- $z$ templates for interpretation of high- $J$ detections in extremely distant galaxies $(z \gtrsim 2)$ with ALMA. Characterizing these transitions relative to the lower ones in the local extreme starburst population-similar to the need to understand local dust SEDs before interpreting the very few frequency points detected for the dust continuum in high-z galaxies-will prove to be extremely valuable before embarking on the high- $J$, high- $z$ ventures with ALMA.

\section{SUMMARY}

The main results presented in this paper are the following.

1. Using the JCMT and IRAM $30 \mathrm{~m}$ telescope, we report first detections of a number of important molecular lines, including transitions of ${ }^{13} \mathrm{CO}, \mathrm{HCN}, \mathrm{CS}$, and $\mathrm{HCO}^{+}$, in the two prototypical ULIRGs Arp 220 and NGC 6240. These observations along with measurements from the literature complete what is currently the largest molecular line catalog for such systems, capable of probing a wide range of densities and temperatures, especially for their important dense and warm star-forming gas phase.

2. In Arp 220, a systematic change in line profile as higher densities are probed suggests large-scale gas-phase segregations within this ULIRG. This is not observed in NGC 6240 even in the profiles of lines widely separated in their excitation requirements such as ${ }^{12} \mathrm{CO} J=2-1$ and $\mathrm{HCN}$ $J=4-3$. This points to the exciting possibility of using high-J/high-dipole molecular lines to deduce the state of gas relaxation in ULIRGs by simply comparing the profiles of lines with widely different excitation requirements. This could prove important since currently only in the closest ULIRGs can interferometry be relied upon to reveal the state of the gas directly. 

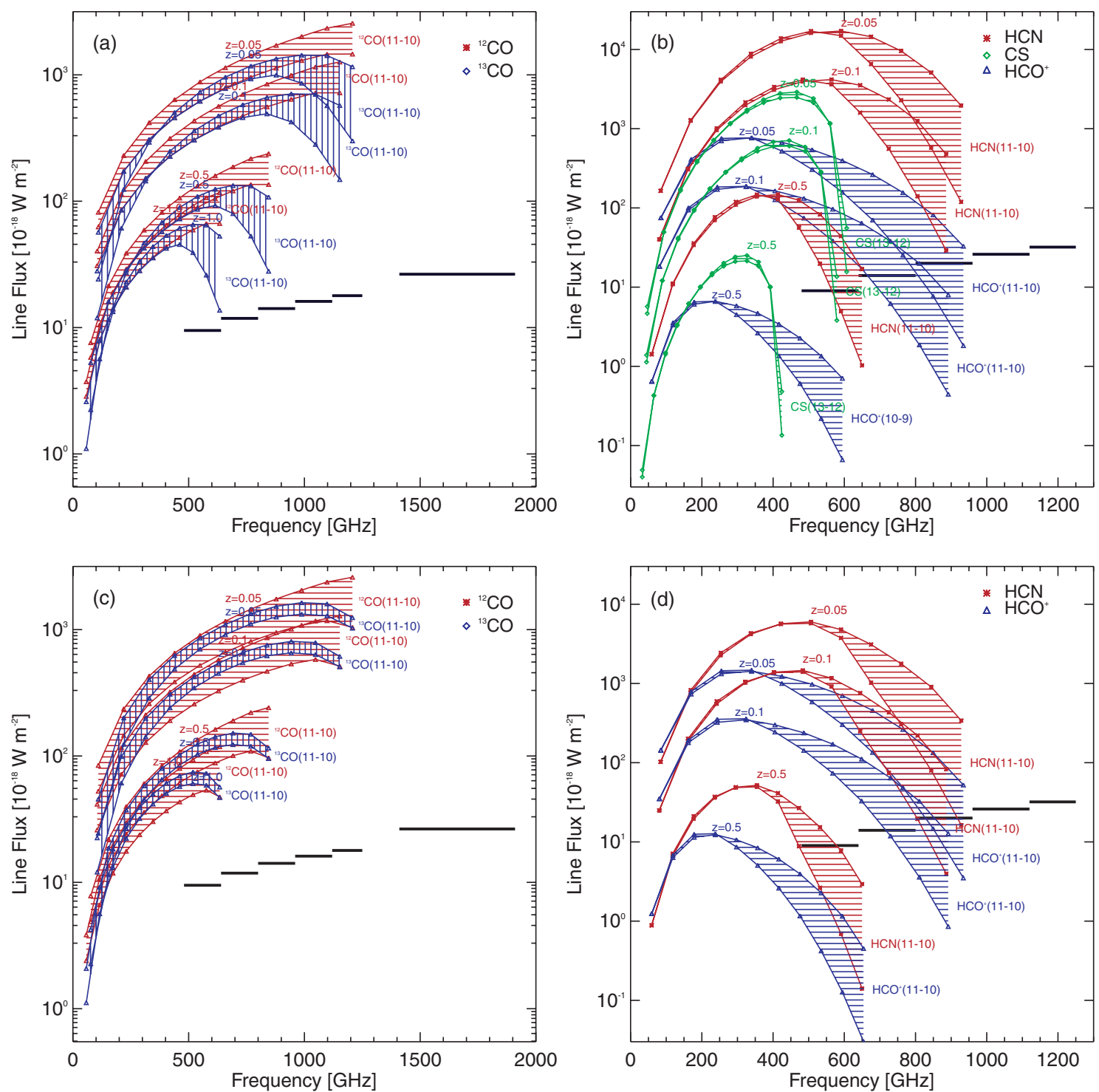

Figure 3. Top panels: integrated line-flux strengths of the full ${ }^{12} \mathrm{CO} /{ }^{13} \mathrm{CO}$ (left) and $\mathrm{HCN} / \mathrm{CS} / \mathrm{HCO}^{+}$(right) rotational ladders predicted by the dense gas template of Arp 220 as given by the solution ranges in Table 7. The line fluxes are shown for Arp 220-like systems at redshifts $z=0.05,0.1$, and 0.5 . In the case of ${ }^{12} \mathrm{CO}$ and ${ }^{13} \mathrm{CO}$, we have also shown the $z=1.0$ template. Bottom panels: Same as above, except the constraints on the dense gas in NGC 6240 have been used as a template. The horizontal bars are the expected $5 \sigma$ sensitivity limits of the five HI-FI bands after $1 \mathrm{~h}$ of integration. In panels (a) and (c), the red and blue curves correspond to the line templates for ${ }^{12} \mathrm{CO}$ and ${ }^{13} \mathrm{CO}$, respectively, while in panels (b) and (d), they correspond to $\mathrm{HCN}$ and $\mathrm{HCO}^{+}$, respectively. The green curve in panel (b) corresponds to the line template for CS.

3. A single molecular gas phase is inadequate to account for the observed properties of the molecular ISM in Arp 220 and NGC 6240. Analysis of the numerous line ratios using a two-phase LVG model finds a diffuse $\left(n\left(\mathrm{H}_{2}\right) \sim\right.$ $10^{2-3} \mathrm{~cm}^{-3}$ ) phase that is most likely unbound, and a dense gas phase $\left(n\left(\mathrm{H}_{2}\right) \sim 10^{5-6} \mathrm{~cm}^{-3}\right)$ that contains the bulk of the molecular gas in these systems $\left(M\left(\mathrm{H}_{2}\right)_{\text {dense }} \sim\right.$ $\left.(1-2) \times 10^{10} \mathrm{M}_{\odot}\right)$.

4. The four highly excited HCN rotational transitions (also four CS transitions for Arp 220) correspond to a gas phase that is $\sim 10-100$ times denser than that suggested by the low, subthermal, $\mathrm{HCO}^{+}$line ratios measured in both systems. Thus HCN trace a denser gas phase than the $\mathrm{HCO}^{+}$lines. Moreover, these two phases are found consistent with an underlying density-size power law, albeit with a steeper exponent than that found in Galactic GMCs (i.e., Larson's law for densities). We argue that this could be another result of the extreme tidal stripping of GMCs in these strongly interacting systems. We also find that for CS all realistic solutions imply $K_{\text {vir }}<<1$, which suggest that the true CS abundance is significantly higher (by an order of magnitude) than the assumed abundance $\left(\left[\mathrm{CS} / \mathrm{H}_{2}\right]=10^{-9}\right)$.

5. Finally, using Arp 220 and NGC 6240 as templates for starburst galaxies at higher redshifts, we show that the HI-FI instrument onboard Herschel will be ideal for detecting very high $J$ transitions of $\mathrm{CO} / \mathrm{HCN} / \mathrm{CS} / \mathrm{HCO}^{+}$in such objects out to redshifts of $z \sim 0.5$ while still preserving the all important information about their relative velocity profiles. Thus the Herschel Space Observatory, in conjunction with an ongoing large millimeter/submillimeter molecular line survey of extreme starbursts, will be able to produce the first densely sampled rotational ladders of $\mathrm{CO}, \mathrm{HCN}$, and $\mathrm{HCO}^{+}$for (U)LIRGs in the local universe, a prerequisite 
in understanding star formation and its relation to dense gas in extreme starbursts locally and in their high-redshift counterparts.

We thank the anonymous referee for a thorough report which helped improve the paper substantially. We are grateful to all the telescope operators at the IRAM $30 \mathrm{~m}$ telescope and the longstanding support we enjoy from the JCMT support personel and telescope operators. We are particular thankful to Jim Hoge at the JCMT for his active role in getting the observations done, often during adverse technical conditions.

\section{REFERENCES}

Aalto, S., Booth, R. S., Black, J. H., \& Johansson, L. E. B. 1995, A\&A, 300 369

Aalto, S., Polatidis, A. G., Hüttemeister, S., \& Curran, S. J. 2002, A\&A, 381 783

Aalto, S., Spaans, M., Wiedner, M. C., \& Hüttemeister, 2007, A\&A, 464, 193

Bayet, E., Gerin, M., Phillips, T. G., \& Contursi, A. 2004, A\&A, 427, 45

Bergin, E. A., Snell, R. L., \& Goldsmith, P. F. 1996, ApJ, 460, 343

Bradford, C. M., Nikola, T., Stacey, G. J., Bolatto, A. D., Jackson, J. M., Savage,

M. L., Davidson, J. A., \& Higdon, S. J. 2003, ApJ, 586, 891

Bryant, P. M., \& Scoville, N. Z. 1996, ApJ, 457, 678

Bryant, P. M., \& Scoville, N. Z. 1999, AJ, 117, 263

Casassus, S., Stahl, O., \& Wilson, T. L. 2005, A\&A, 441, 181

Casoli, F, Dupraz, C., \& Combes, F. 1992, A\&A, 264, 55

de Graauw, T., et al. 1998, Proc. SPIE, 3357, 336

de Graauw, T., et al. 2005, BAAS, 37, 1219

Dickman, R. L., Snell, R. L., \& Schloerb, F. P. 1986, ApJ, 309, 326

Dopita, M. A., et al. 2005, ApJ, 619, 755

Downes, D., \& Solomon, P. M. 1998, ApJ, 507, 615

Flower, D. R. 1999, MNRAS, 305, 651

Flower, D. R., \& Launay, J. M. 1985, MNRAS, 214, 271

Gao, Y., \& Solomon, P. M. 2004a, ApJS, 152, 63

Gao, Y., \& Solomon, P. M. 2004b, ApJ, 606, 271

Goldsmith, P. F. 2001, ApJ, 557, 736

Goldsmith, P. F., \& Langer, W. D. 1978, ApJ, 222, 881

Goldsmith, P. F., Langer, W. D., Ellder, J., Kollberg, E., \& Irvine, W. 1981, ApJ, 249,524

Gordon, M. A., Baars, J. W. M., \& Cocke, W. J. 1992, A\&A, 264, 337

Graciá-Carpio, J., García-Burillo, S., Planesas, P., \& Colina, L. 2006, ApJ, 640, L135

Green, S., \& Thaddeus, P. 1974, ApJ, 191, 653

Hatchell, J., \& van der Tak, F. F. S. 2003, A\&A, 409, 589

Heyer, M. H., \& Brunt, C. M. 2004, ApJ, 615, L45

Hüttemeister, S., Henkel, C., Mauersberger, R., Brouillet, N., Wiklind, T., \& Millar, T. J. 1995, A\&A, 295, 571

Iwasawa, K., Sanders, D. B., Evans, A. S., Trentham, N., Miniutti, G., \& Spoon, H. W. W. 2005, MNRAS, 357, 565

Jackson, J. M., Paglione, T. A. D., Carlstrom, J. E., \& Nguyen, Q. R. 1995, ApJ, 438, 695

Jansen, D. 1995, PhD thesis, Sterrewacht, Universiteit Leiden

Komossa, S., Burwitz, V., Hasinger, G., Predehl, P., Kaastra, J. S., \& Ikebe, Y. 2003, ApJ, 582, L15

Krips, M., et al. 2008, ApJ, 677, 262

Krumholz, M. R., \& McKee, C. F. 2005, ApJ, 630, 250

Kutner, M. L., \& Ulich, B. L. 1981, ApJ, 250, 341
Lahuis, F., \& van Dishoeck, E. F. 2000, A\&A, 355, L699

Langer, W. D., \& Penzias, A. A. 1993, ApJ, 408, 539

Larson, R. B. 1981, MNRAS, 194, 809

Lepp, S., \& Dalgarno, A. 1996, A\&A, 306, L21

Lisenfeld, U., Hills, R. E., Radford, S. J. E., \& Solomon, P. M. 1996, Cold Gas at High Redshifts, (Dordrecht : Kluwer), 55

Lonsdale, C., Farrah, D., \& Smith, H. 2006, in Astrophysics Update 2-Topical and Timely Reviews on Astronomy and Astrophysics, ed. John W. Mason, (Chichester: Springer, Praxis Books)

Mauersberger, R., Henkel, C., Walsh, W., \& Schulz, A. 1999, A\&A, 341, 256

Nakanishi, K., Okumura, S. K., Kohno, K., Kawabe, R., \& Nakagaw, T. 2005, PASJ, 57, 575

Nguyen, Q-Rieu, Jackson, J. M., Henkel, C., Truong, B., \& Mauersberger, R. 1992, ApJ, 399, 521

Paglione, T. A. D., Jackson, J. M., \& Ishizuki, S. 1997, ApJ, 484, 656

Paglione, T. A. D., et al. 1995, AJ, 109, 1716

Papadopoulos, P. P. 2007, ApJ, 656, 792

Papadopoulos, P. P., \& Greve, T. R. 2004, ApJ, 615, L29

Papadopoulos, P. P., Greve, T. R., van der Werf, P. P., Müehle, S., Isaak, K., \& Gao, Y. 2007a, Ap\&SS (arXiv:astro-ph/0701829)

Papadopoulos, P. P., Isaak, K. G., \& van der Werf, P. P. 2007b, ApJ, 668, 815

Papadopoulos, P. P., \& Seaquist, E. R. 1998, ApJ, 492, 521

Papadopoulos, P. P., \& Seaquist, E. R. 1999, ApJ, 516, 114

Papadopoulos, P. P., Seaquist, E. R., \& Scoville, N. Z. 1996, ApJ, 465, 173

Plume, R., Jaffe, D. T., Evans, N. J., Martín-Pintado, J., \& Gómez-González, J. 1997, ApJ, 476, 730

Radford, S. J. E., Solomon, P. M., \& Downes, D. 1991a, ApJ, 368, L15

Radford, S. J. E., et al. 1991b, IAU Symp. 146 (Dordrecht: Kluwer) 303

Richardson, K. J. 1985, PhD Thesis, Department of Physics, Queen Mary College, Univ. London

Sakamoto, K., et al. 2008, ApJ, 684, 957

Sanders, D. B., \& Mirabel, I. F. 1996, ARA\&A, 34, 749

Schilke, P., Walmsley, C. M., Pineau Des Forets, G., Roueff, E., Flower, D. R. \& Guilloteau, S. 1992, A\&A, 256, 595

Scoville, N. Z. 2004, in Proc. The Neutral ISM in Starburst Galaxies, Marstrand, Sweden, ed. S. Aalto, S. Hüttemeister, \& A. Pedlar, 253

Scoville, N. Z., Sargent, A. I., Sanders, D. B., \& Soifer, B. T. 1991, ApJ, 366, L5

Scoville, N. Z., Yun, M. S., \& Bryant, P. M. 1997, ApJ, 484, 702

Seaquist, E. R., Lee, S. W., \& Moriarty-Schieven, G. H. 2006, ApJ, 638, 148

Shirley, Y. L., Evans, N. J., Young, K. E., Knez, C., \& Jaffe, D. T. 2003, ApJS, 149,375

Soifer, B. T., Sanders, D. B., Neugebauer, G., Danielson, G. E., Lonsdale, C. J., Madore, B. F., \& Persson, S. E. 1986, ApJ, 303, L41

Solomon, P. M., Downes, D., \& Radford, S. J. E. 1992a, ApJ, 387, L55

Solomon, P. M., Downes, D., \& Radford, S. J. E. 1992b, ApJ, 398, L29

Solomon, P. M., Downes, D., Radford, S. J. E., \& Barrett, J. W. 1997, ApJ, 478 144

Solomon, P. M., Radford, S. J. E., \& Downes, D. 1990, ApJ, 348, L53

Spergel, D. N., et al. 2003, ApJS, 148, 175

Tacconi, L. J., Genzel, R., Tecza, M., Gallimore, J. F., Downes, D., \& Scoville, N. Z. 1999, ApJ, 524, 732

Turner, B. E., Chan, K, Green, S., \& Lubowich, D. A. 1992, ApJ, 399, 114

Usero, A., García-Burillo, S., Fuente, A., \& Martín-Pintado, J. 2004, ASP Conf. Ser. 320 (San Francisco, CA: ASP), 273

Weiß, A., Walter, F., \& Scoville, N. Z. 2005, A\&A, 438, 533

Wiedner, M. C., Wilson, C. D., Harrison, A., Hills, R. E., Lay, O. P., \& Carlstrom, J. E. 2002, ApJ, 581, 299

Wolfire, M. G., McKee, C. F., Hollenbach, D., \& Tielens, A. G. G. M. 2003, ApJ, 587,278

Wu, J., Evans, N. J., Gao, Y., Solomon, P. M., Shirley, Y. L., \& Vanden Bout, P. A. 2005, ApJ, 635, L173 